ESAIM: M2AN 50 (2016) 879-904

DOI: $10.1051 / \mathrm{m} 2 \mathrm{an} / 2015090$
ESAIM: Mathematical Modelling and Numerical Analysis

www.esaim-m2an.org

\title{
THE NONCONFORMING VIRTUAL ELEMENT METHOD
}

\author{
Blanca Ayuso de Dios ${ }^{1,2}$, Konstantin Lipnikov ${ }^{3}$ And Gianmarco Manzini ${ }^{2,3}$
}

\begin{abstract}
We introduce the nonconforming Virtual Element Method (VEM) for the approximation of second order elliptic problems. We present the construction of the new element in two and three dimensions, highlighting the main differences with the conforming VEM and the classical nonconforming finite element methods. We provide the error analysis and establish the equivalence with a family of mimetic finite difference methods. Numerical experiments verify the theory and validate the performance of the proposed method.
\end{abstract}

Mathematics Subject Classification. 65N30, 65N12, 65G99, 76R99.

Received April 26, 2015. Revised November 2, 2015.

Published online May 23, 2016.

\section{INTRODUCTION}

Methods that can handle general meshes consisting of arbitrary polygons or polyhedra have received significant attention over the last years. Among them the Mimetic Finite Difference (MFD) method [6] that has been successfully applied to a wide range of scientific and engineering applications (see, for instance, $[17,31,32,37]$ and the references therein). However, the construction of high-order MFD schemes is still a challenging task even for two- and three-dimensional second-order elliptic problems. For example, the two-dimensional MFD scheme in [5] could be seen as the high-order extension of the lower-order scheme given in [15]. A straightworward extension of [5] to three dimensions would lead to a clumsy discretization involving a huge number of degrees of freedom that ensure conformity of the approximation. By relaxing the conformity condition, a simpler MFD scheme has been proposed in [30] for three dimensional elliptic problems. Lately, there have been introduced other relevant approaches for handling general meshes [22-24].

Very recently, in the pioneering work [7], the basic principles of the virtual element method (VEM) have been introduced. The VEM allows one to recast the MFD schemes $[5,15]$ as Galerkin formulations. The virtual element methodology generalizes the "classical" finite element method to mesh partitions consisting of polygonal and polyhedral elements of very general shapes including non-convex elements. In this respect, it shares with the MFD method the flexibility of mesh handling. Unlike the MFD method, the VEM provides a sound mathematical framework that allows to devise and analyze new schemes in a much simpler and elegant way. The name virtual

\footnotetext{
Keywords and phrases. Virtual element method, nonconforming method, Poisson equation, elliptic problems, unstructured meshes.

1 Institut für Mathematik, Technische Universität Hamburg-Harburg, Am Schwarzenberg-Campus 3, D-21073 Hamburg, Germany. blanca.ayuso@tuhh.de

2 Istituto di Matematica Applicata e Tecnologie Informatiche (IMATI) - CNR, 27100 Pavia, Italy. marco.manzini@imati.cnr.it

3 Applied Mathematics and Plasma Physics Group, Theoretical Division, Los Alamos National Laboratory, Los Alamos, NM 87545, USA. lipnikov@lanl.gov; gmanzini@lanl.gov
} 
comes from the fact that the local approximation space in each mesh polygon or polyhedron contains a space of polynomials together with some other functions that are solutions of particular partial differential equations. Such functions are never computed and similar to the MFD method, the VEM can be implemented using only the degrees of freedom and the polynomial part of the approximation space. We refer to [9] for the implementation details.

Despite of its infancy, the conforming VEM laid in [7] has been already extended to a variety of two dimensional problems: plate problems are studied in [13], linear elasticity in [8], mixed methods for $H$ (div; $\Omega$ )approximations are introduced in [16], and very recently the VEM has been extended to simulations on discrete fracture networks [10]. In [2], further tools are presented that allow us to construct and analyze the conforming VEM for three dimensional elliptic problems. The definition of the three dimensional virtual element spaces in [2], requires the use of the two dimensional ones.

In this paper, we develop and analyze the nonconforming VEM for the approximation of second order elliptic problems in two and three dimensions. We show that the proposed method contains the MFD schemes from [30]. In contrast to the conforming VEM, our construction is done simultaneously for any dimension and any approximation order. To put this work in perspective, we present below a brief (non exhaustive) overview of nonconforming finite element methods.

\subsection{Overview of nonconforming finite element methods}

Nonconforming finite elements were first recognized as a variational crime; a term first coined by Strang in $[40,41]$. In the case of second order elliptic problems, the approximation space has some continuity built in it, but still discrete functions are not continuous. Still, that relaxed continuity (or crime) has proved its usefulness in many applications, mostly related to continuum mechanics, in particular, for fluid flow problems $[29,38]$ (for moderate Reynolds numbers) and elasticity $[25,36]$.

The construction, analysis and understanding of nonconforming elements have received much attention since their first introduction for second order elliptic problems. In two dimensions, the design of schemes of order of accuracy $k \geq 1$ was guided by the patch-test, which enforces continuity at $k$ Gauss-Legendre points on edge. Due to different behavior of odd and even polynomials, the construction of schemes for odd and even $k$ is different, with the latter case demanding much more elaborated arguments. Furthermore, the shape of the elements (triangular or rectangular/quadrilateral) adds additional complexity to the construction of nonconforming elements [38] (the result of having an odd or even number of edges in the element leads to a different construction). For the Stokes problem with the Dirichlet boundary conditions, Crouzeix and Raviart proposed and analyzed the first order $(k=1)$ nonconforming finite element approximation of the velocity field in [20], which is now know as the Crouziex-Raviart element. The extension to degree $k=3$ was given in [21], while the construction for degree $k=2$ was introduced in [27]. In all cases, the inf-sup stable Stokes pair is formed by considering discontinuous approximation for the pressure of one degree lower.

Already in the 80 's, an equivalence between mixed methods and a modified version of nonconforming elements of odd degree has been established in [3,33] and exploited in the analysis and implementation of the methods. The author of [19], inspired by [3], has studied the hybridization of the mixed Hellan-Herrmann-Johnson method (of any degree) for the approximation of a fourth order problem. As a byproduct of the analyzed postprocessing technique (that uses the gradient of the displacement which would play the role of the velocity field in the Stokes problem), a construction of nonconforming elements of any degree $k$ is provided. Again, this construction distinguishes between odd and even degrees. Although the details are for the fourth order problem, the strategy can be adapted to other elliptic problems. For $k=1,3$, the nonconforming element coincides with the construction given in $[20,21]$ for the Stokes problem. For even $k$, in addition to the moments of order $k-1$ on each edge, an extra degree of freedom is required to ensure unisolvence. In the case $k=2$ the resulting nonconforming local finite element space has the same dimension but is different to the one proposed in [27] where it is constructed by adding a nonconforming bubble to the second order conforming space.

Over the last years, further generalizations of the nonconforming elements have been still considered by several authors; always distinguishing between odd and even degrees. In [4,39] a construction similar to the one 
given in [19] is considered for the Stokes problem. A rather different approach is considered in [34]. Finally, while the extension to three dimensions is simple for $k=1$, already for $k=2$, the construction of a nonconforming element becomes cumbersome [26].

\subsection{Main contributions}

In this paper, we extend the virtual element methodology by developing in one-shot (no special cases) a nonconforming approximation of any degree for any spatial dimension and any element shape. For triangular meshes and $k=1,2$, the proposed nonconforming VEM has the same degrees of freedom as the related nonconforming finite element in [19]. For quadrilaterals and $k=1$, the degrees of freedom are the same as that in [38]. The three main contributions of the present work are as follows.

(i) The nonconforming VEM is constructed for any order of accuracy and for arbitrarily-shaped polygonal or/and polyhedral elements. It also provides a simpler construction on simplicial meshes and quadrilateral meshes.

(ii) Unlike the conforming VEM [2], the nonconforming VEM is introduced and analyzed at once for two and three dimensional problems. This simplifies substantially its analysis and practical implementation.

(iii) We prove optimal error estimates in the energy norm and (for $k \geq 2$ ) in the $L^{2}$-norm. The analysis of the new method is carried out using techniques already introduced in $[7,8]$ and extending the results well known in the finite element method to the virtual approach. As the byproduct of our analysis, we provide the theory for the MFD schemes in [30].

To convey the main idea of our work in a better way and to keep the presentation simple, we consider the Poisson problem with a constant diffusion tensor. However, all results apply (with minor changes) to more general second order problems with smoothly varying coefficients. This latter case will be addressed in the section of numerical experiments.

The outline of the paper is as follows. In Section 2 we formulate the problem and introduce the basic setting. In Section 3 we introduce the nonconforming VEM. Section 4 is devoted to the error analysis of the nonconforming approximation. In Section 5 we establish the connection with the nonconforming MFD method proposed in [30]. In Section 6 we show the performance of the method in numerically solving the Poisson problem with constant and variable diffusion coefficients. In Section 7 we offer some final remarks and discuss the perspectives for future work and developments.

\section{Continuous Problem AND BASiC SETting}

In this section we present the basic setting and describe the continuous problem.

Notation: Throughout the paper, we use the standard notation of Sobolev spaces, $c f$. [1]. Moreover, for any integer $\ell \geq 0$ and a domain $D \in \mathbb{R}^{m}$ with $m \leq d, d=2,3, \mathbb{P}^{\ell}(D)$ is the space of polynomials of degree at most $\ell$ defined on $D$. We also adopt the convention that $\mathbb{P}^{-1}(D)=\{0\}$.

\subsection{Continuous problem}

Let the domain $\Omega$ in $\mathbb{R}^{d}$ with $d=2,3$ be a bounded open polytope with boundary $\partial \Omega$, e.g., a polygonal domain with straight boundary edges for $d=2$ or a polyhedral domain with flat boundary faces for $d=3$. Let $f$ be in $L^{2}(\Omega)$ and consider the model problem:

$$
\begin{aligned}
-\Delta u=f & \text { in } \Omega, \\
u=g & \text { on } \partial \Omega .
\end{aligned}
$$

Let $V_{g}=\left\{v \in H^{1}(\Omega):\left.v\right|_{\partial \Omega}=g\right\}$ and $V=H_{0}^{1}(\Omega)$. The variational formulation of problem (2.1) and (2.2) reads as:

$$
\text { Find } u \in V_{g} \text { such that: } a(u, v)=\langle f, v\rangle \quad \forall v \in V \text {, }
$$


where the bilinear form $a: V \times V \rightarrow \mathbb{R}$ is given by

$$
a(u, v)=\int_{\Omega} \nabla u \cdot \nabla v \mathrm{~d} x \quad \forall u, v \in V,
$$

and $\langle\cdot, \cdot\rangle$ denotes the duality product between the functional spaces $V^{\prime}$ and $V$. The bilinear form in (2.4) is continuous and coercive with respect to the $H_{0}^{1}$-seminorm (which is a norm in $V$ by Poincaré inequality); therefore, the Lax-Milgram theorem ensures the well posedness of the variational problem and the existence of a unique solution $u \in V$ to $(2.3)$.

\subsection{Basic setting}

We describe now the basic assumptions of the mesh partitioning and introduce some further functional spaces.

Let $\left\{\mathcal{T}_{h}\right\}_{h}$ be a family of decompositions of $\Omega$ into elements $K$ and let $\mathcal{E}_{h}$ denote the skeleton of the partition, i.e., the set of edges/faces of $\mathcal{T}_{h}$. By $\mathcal{E}_{h}^{o}$ and $\mathcal{E}_{h}^{\partial}$ we will refer to the set of interior and boundary edges/faces, respectively. Following $[2,7]$ we make the following assumptions on the family of partitions:

(A0) Assumptions on the family of partitions $\left\{\mathcal{T}_{h}\right\}_{h}$ : we assume that there exists a positive $\varrho>0$ such that

- for every element $K$ and for every edge/face $e \subset \partial K$, we have: $h_{e} \geq \varrho h_{K}$,

- every element $K$ is star-shaped with respect to all the points of a sphere of radius $\geq \varrho h_{K}$;

- for $d=3$, every face $e \in \mathcal{E}_{h}$ is star-shaped with respect to all the points of a disk having radius $\geq \varrho h_{e}$.

The maximum of the diameters of the elements $K \in \mathcal{T}_{h}$ will be denoted by $h$. For every $h>0$, the partition $\mathcal{T}_{h}$ is made of a finite number of polygons or polyhedra.

We introduce the broken Sobolev space for any $s>0$

$$
H^{s}\left(\mathcal{T}_{h}\right)=\prod_{K \in \mathcal{T}_{h}} H^{s}(K)=\left\{v \in L^{2}(\Omega):\left.v\right|_{K} \in H^{s}(K)\right\}
$$

and define the broken $H^{s}$-norm

$$
\|v\|_{s, \mathcal{T}_{h}}^{2}=\sum_{K \in \mathcal{T}_{h}}\|v\|_{s, K}^{2} \quad \forall v \in H^{s}\left(\mathcal{T}_{h}\right)
$$

and for $s=1$ the broken $H^{1}$-seminorm

$$
|v|_{1, h}^{2}=\sum_{K \in \mathcal{T}_{h}}\|\nabla v\|_{0, K}^{2} \quad \forall v \in H^{1}\left(\mathcal{T}_{h}\right)
$$

When $s=0$ we will prefer the notation $\|v\|_{0, \Omega}$ instead of $\|v\|_{s, \mathcal{T}_{h}}$. Let $e \subset \partial K^{+} \cap \partial K^{-}$be an edge/face in $\mathcal{E}_{h}^{o}$. For $v \in H^{1}\left(\mathcal{T}_{h}\right)$, by $v^{ \pm}$we denote the trace of $\left.v\right|_{K^{ \pm}}$on $e$ taken from within the element $K^{ \pm}$and by $n_{e}^{ \pm}$we denote the unit normal on $e$ in the outward direction with respect to $K^{ \pm}$. We then define the jump operator as:

$$
\llbracket v \rrbracket=v^{+} \mathbf{n}_{e}^{+}+v^{-} \mathbf{n}_{e}^{-} \quad \text { on } e \in \mathcal{E}_{h}^{o} \quad \text { and } \quad \llbracket v \rrbracket=v \mathbf{n}_{e} \text { on } e \in \mathcal{E}_{h}^{\partial},
$$

where on boundary edges/faces we have defined it as the normal component of the trace of $v$.

It is convenient to introduce a subspace of $H^{1}\left(\mathcal{T}_{h}\right)$ with some continuity built in. For any integer $k \geq 1$, we define

$$
H^{1, \mathrm{nc}}\left(\mathcal{T}_{h} ; k\right)=\left\{v \in H^{1}\left(\mathcal{T}_{h}\right): \int_{e} \llbracket v \rrbracket \cdot \mathbf{n}_{e} q \mathrm{~d} s=0 \forall q \in \mathbb{P}^{k-1}(e), \forall e \in \mathcal{E}_{h}\right\} .
$$


Although for discontinuous functions $|\cdot|_{1, h}$ is only a semi-norm, for $v \in V$ and $v \in H^{1, \mathrm{nc}}\left(\mathcal{T}_{h}\right)$ it is indeed a norm. In fact, a standard application of the results in [11] shows that a Poincaré inequality holds for functions in $H^{1, n c}\left(\mathcal{T}_{h}\right)$ (already with $k=1$ ), i.e., there exists a constant $C_{P}>0$ independent of $h$ such that

$$
\|v\|_{0, \Omega}^{2} \leq C_{P}|v|_{1, h}^{2} \quad \forall v \in H^{1, \mathrm{nc}}\left(\mathcal{T}_{h}\right) .
$$

Therefore, with a small abuse of notation we will refer to the broken semi-norm as a norm.

Remark 2.1. The space $H^{1, \mathrm{nc}}\left(\mathcal{T}_{h} ; 1\right)(i . e ., k=1)$, is the space with minimal required continuity to ensure that the analysis can be carried out.

Finally, the bilinear form $a(\cdot, \cdot)$ can be split as:

$$
a(u, v)=\sum_{K \in \mathcal{T}_{h}} a^{K}(u, v) \quad \text { where } \quad a^{K}(u, v)=\int_{K} \nabla u \cdot \nabla v \mathrm{~d} x \quad \forall u, v \in V .
$$

\section{NONCONFORMing VIRTUAL ELEMENT METHOD}

In this section we introduce the nonconforming virtual element method for the model problems (2.1) and (2.2), which we write as the Galerkin approximation:

$$
\text { Find } u_{h} \in V_{h, g}^{k} \text { such that: } a_{h}\left(u_{h}, v_{h}\right)=\left\langle f_{h}, v_{h}\right\rangle \quad \forall v_{h} \in V_{h}^{k} \text {, }
$$

where $V_{h}^{k} \subset H^{1, \mathrm{nc}}\left(\mathcal{T}_{h} ; k\right)$ is the global nonconforming virtual element space; $V_{h, g}^{k}$ is the affine space required by the numerical treatment of the Dirichlet boundary conditions; $a_{h}(\cdot, \cdot)$ and $\left\langle f_{h}, \cdot\right\rangle$ are the nonconforming approximation to the bilinear form $a(\cdot, \cdot)$ and the linear functional $\langle f, \cdot\rangle$, respectively.

We start by describing the local and global nonconforming virtual element spaces, denoted by $V_{h}^{k}(K)$ and $V_{h}^{k}$, respectively. We then construct the discrete bilinear form $a_{h}(\cdot, \cdot)$ and the forcing term $f_{h}$, discussing also their main properties for the analysis of the resulting approximation. Through the whole section, we follow the basic ideas given in $[2,7]$, trying to highlight the main differences with the present case.

\subsection{The local nonconforming virtual element space $V_{h}^{k}(K)$}

We need to introduce some further notation. For a polygon or polyhedron $K$ with $n$ edges/faces we denote by $x_{K}$ its center of gravity, by $|K|$ its $d$-dimensional measure (area for $d=2$, volume for $d=3$ ) and by $h_{K}$ its diameter. Similarly, for each edge/face $e \subset \partial K$, we denote by $x_{e}$ its midpoint/barycenter, by $|e|$ its measure and by $h_{e}$ its diameter. As before, $\mathbf{n}_{K}$ denotes the outward unit normal on $\partial K$ and $\mathbf{n}_{e}$ refers to the unit vector normal to $e$, whose orientation is fixed once and for all.

For $k \geq 1$, we define the local non-conforming virtual element space of order $k$ associated with the polygon/polyhedron $K$ as the finite dimensional space

$$
V_{h}^{k}(K)=\left\{v \in H^{1}(K): \frac{\partial v}{\partial \mathbf{n}} \in \mathbb{P}^{k-1}(e) \forall e \subset \partial K, \Delta v \in \mathbb{P}^{k-2}(K)\right\},
$$

with the usual convention that $\mathbb{P}^{-1}(K)=\{0\}$.

For $k=1$, the local virtual element space $V_{h}^{1}(K)$ consists of functions $v$ for which the normal derivative $\frac{\partial v}{\partial \mathbf{n}}$ is constant (and possibly different) on each $e \subset \partial K$ and that are harmonic inside $K$, i.e., $\Delta v=0$. The dimension of $V_{h}^{1}(K)$ is equal to $n$, the number of edges/faces of $K$.

For $k=2$, the space $V_{h}^{2}(K)$ consists of functions $v$ for which the normal derivative along the edges/faces $e \in \partial K$ is a linear polynomial and, inside $K$, are such that $\Delta v$ is constant. A simple counting reveals that the dimension of $V_{h}^{2}$ is $d n+1$. 


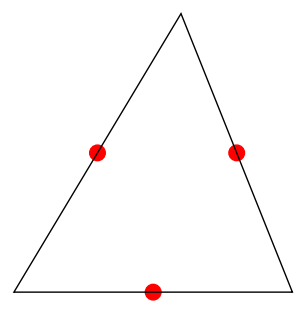

$\mathrm{k}=1$

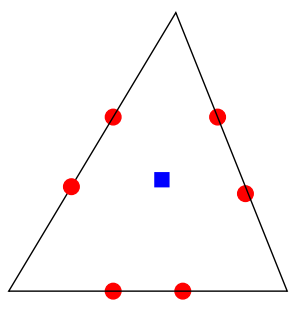

$\mathrm{k}=\mathbf{2}$

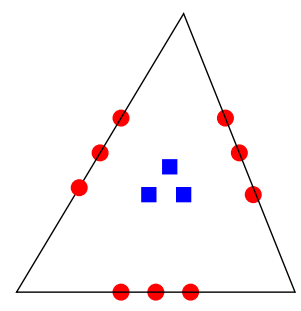

$\mathrm{k}=3$

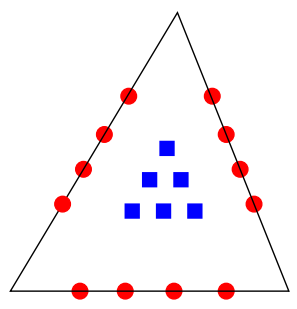

$\mathrm{k}=4$

Figure 1. Degrees of freedom of a triangular cell for $k=1,2,3,4$; edge moments are marked by a circle; cell moments are marked by a square.

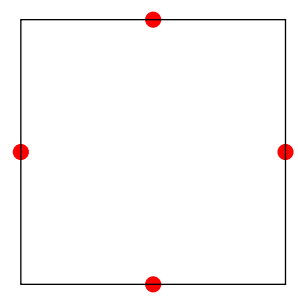

$\mathrm{k}=1$

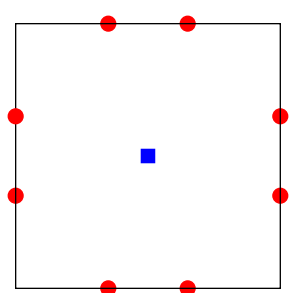

$\mathrm{k}=2$

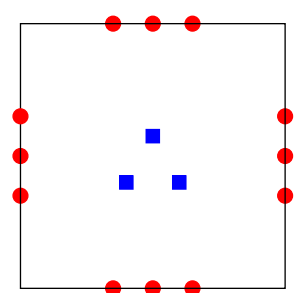

$\mathrm{k}=3$

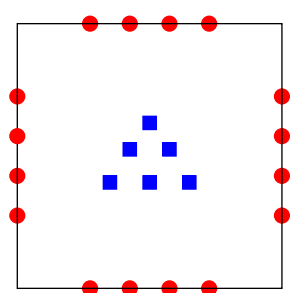

$\mathrm{k}=4$

FiguRE 2. Degrees of freedom of a quadrilateral cell for $k=1,2,3,4$; edge moments are marked by a circle; cell moments are marked by a square.

For each polygon/polyhedron $K$, the dimension of $V_{h}^{k}(K)$ is given by

$$
N_{K}= \begin{cases}n k+(k-1) k / 2 & \text { for } d=2, \\ n k(k+1) / 2+(k-1) k(k+1) / 6 & \text { for } d=3 .\end{cases}
$$

Let $s=\left(s_{1}, \ldots, s_{d}\right)$ be a $d$-dimensional multi-index with the usual notation that $|s|=\sum_{i=1}^{d} s_{i}$ and $\mathbf{x}^{s}=$ $\prod_{i=1}^{d} x_{i}^{s_{i}}$ where $\mathbf{x}=\left(x_{1}, \ldots, x_{d}\right) \in \mathbb{R}^{d}$. For $\ell \geq 0$, the symbols $\mathcal{M}^{\ell}(e)$ and $\mathcal{M}^{\ell}(K)$ denote the set of scaled monomials on $e$ and $K$ :

$$
\mathcal{M}^{\ell}(e)=\left\{\left(\frac{\mathbf{x}-\mathbf{x}_{e}}{h_{e}}\right)^{s},|s| \leq \ell\right\} \quad \text { and } \quad \mathcal{M}^{\ell}(K)=\left\{\left(\frac{\mathbf{x}-\mathbf{x}_{K}}{h_{K}}\right)^{s},|s| \leq \ell\right\} .
$$

In $V_{h}^{k}(K)$ we can choose the following degrees of freedom:

(i) all the moments of $v_{h}$ of order up to $k-1$ on each edge/face $e \in \partial K$ :

$$
\mu_{e}^{k-1}\left(v_{h}\right)=\left\{\frac{1}{|e|} \int_{e} v_{h} m \mathrm{~d} s, \quad \forall m \in \mathcal{M}^{k-1}(e)\right\} \forall e \subset \partial K
$$

(ii) all the moments of $v_{h}$ of order up to $k-2$ on $K$ :

$$
\mu_{K}^{k-2}\left(v_{h}\right)=\left\{\frac{1}{|K|} \int_{K} v_{h} m \mathrm{~d} x, \quad \forall m \in \mathcal{M}^{k-2}(K)\right\} .
$$

For $k=1,2,3,4$, the degrees of freedom are shown for a triangular, a quadrilateral and an hexagonal element in Figures 1-3, and for a tetrahedral and a cubic element in Figures 4 and 5. 


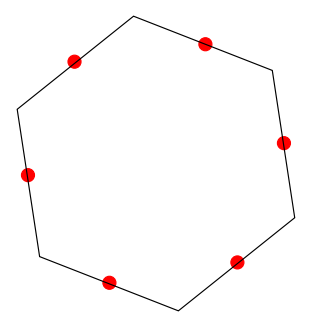

$\mathrm{k}=1$

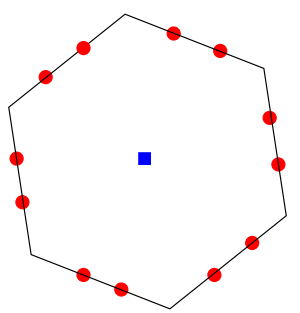

$\mathrm{k}=2$

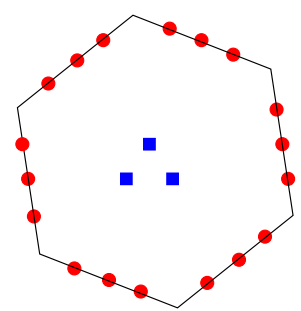

$\mathrm{k}=3$

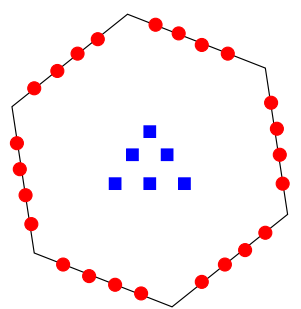

$\mathrm{k}=4$

FiguRE 3. Degrees of freedom of a hexagonal cell for $k=1,2,3,4$; edge moments are marked by a circle; cell moments are marked by a square.

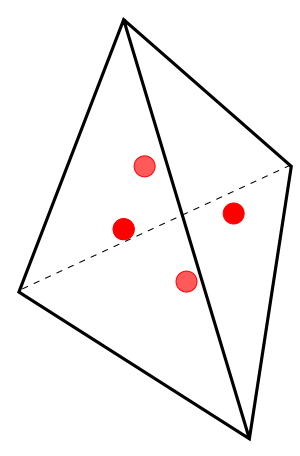

$\mathrm{k}=1$

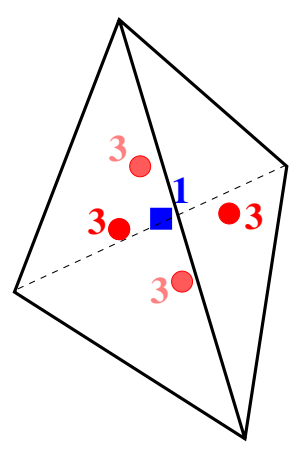

$\mathrm{k}=\mathbf{2}$

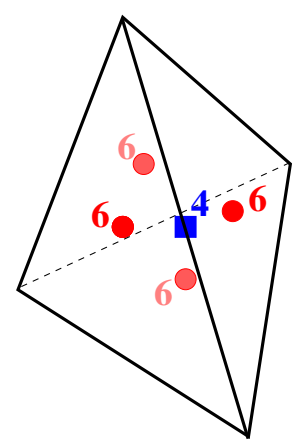

$\mathrm{k}=3$

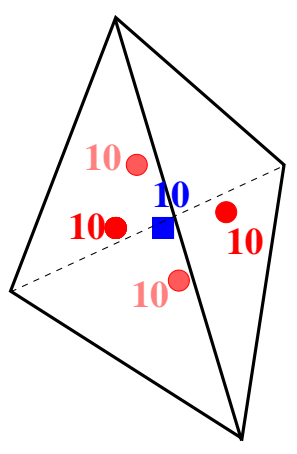

$\mathrm{k}=4$

FiguRE 4. Degrees of freedom of a tetrahedral cell for $k=1,2,3$, 4; face moments are marked by a circle; cell moments are marked by a square. The numbers indicates the number of degrees of freedom ( 1 is not marked in the plot for $k=1$ ).

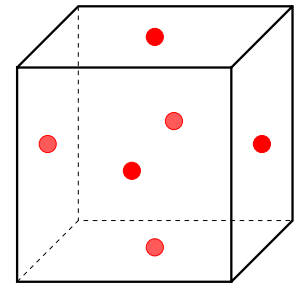

$\mathrm{k}=1$

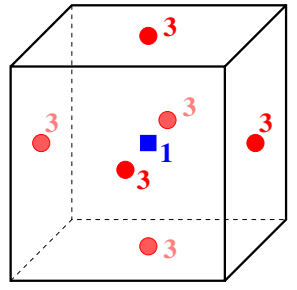

$\mathrm{k}=2$

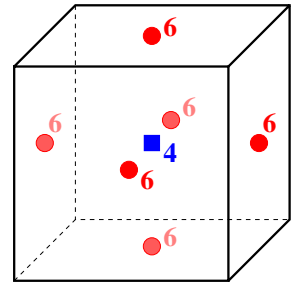

$\mathrm{k}=3$

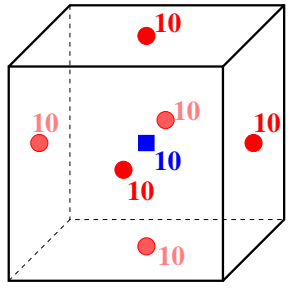

$\mathrm{k}=4$

Figure 5. Degrees of freedom of a cubic cell for $k=1,2,3,4$; face moments are marked by a circle; cell moments are marked by a square. The numbers indicate the number of degrees of freedom ( 1 is not marked in the plot for $k=1$ ).

Observe that the dimension $N_{K}$ given by (3.3) coincides with the total number of degrees of freedom defined in (3.5) and (3.6). They are indeed unisolvent for the local space $V_{h}^{k}(K)$ as we show next:

Lemma 3.1. Let $K$ be a simple polygon/polyhedron with $n$ edges/faces, and let $V_{h}^{k}(K)$ be the space defined in (3.2) for any integer $k \geq 1$. The degrees of freedom (3.5) and (3.6) are unisolvent for $V_{h}^{k}(K)$. 
Proof. Notice, that we cannot proceed as for the unisolvence proofs in finite elements, since $V_{h}^{k}(K)$ would typically contain functions that are not polynomial. Still, we need to show that for any $v_{h} \in V_{h}^{k}(K)$ such that

$$
\mu_{e}^{k-1}\left(v_{h}\right)=0 \quad \forall e \subset \partial K \quad \text { and } \quad \mu_{K}^{k-2}\left(v_{h}\right)=0
$$

then $v_{h}=0$. To do so, we use the Divergence Theorem (with $v_{h} \in V_{h}^{k}(K)$ and so $\frac{\partial v_{h}}{\partial \mathbf{n}} \in \mathbb{P}^{k-1}(e)$ on each $e \subset \partial K$ and $\left.\Delta v \in \mathbb{P}^{k-2}(K)\right)$ to get

$$
\int_{K}\left|\nabla v_{h}\right|^{2} \mathrm{~d} x=-\int_{K} v_{h} \Delta v_{h} \mathrm{~d} x+\sum_{e \in \partial K} \int_{e} v_{h} \frac{\partial v_{h}}{\partial \mathbf{n}} \mathrm{d} s=0,
$$

where we have set the right hand side equal to zero using the fact that the degrees of freedom of $v_{h}$ vanish from (3.7). Hence $\nabla v_{h}=0$ in $K$ and so $v_{h}=$ constant in $K$. But, since $\mu_{e}^{0}\left(v_{h}\right)=0$ (the zero-order moment on each $e \subset \partial K$ vanishes), we deduce that $v_{h}=0$ in $K$.

Remark 3.2. The degrees of freedom of the method (3.5) and (3.6) are defined by using the monomials in $\mathcal{M}^{k-1}(e)$ and $\mathcal{M}^{k-2}(K)$ as basis functions for the polynomial spaces $\mathbb{P}^{k-1}(e)$ and $\mathbb{P}^{k-2}(K)$. This special choice of the basis functions gives the method an inherent hierarchical structure with respect to $k$, which may be useful for an efficient implementation. However, the construction of the element is independent of such choice and, in principle, any other basis (properly defined and scaled) could be used to define the degrees of freedom.

\subsection{The global nonconforming virtual element space $V_{h}^{k}$}

We now introduce the nonconforming (global) virtual element space $V_{h}^{k}$ of order $k$. For every decomposition $\mathcal{T}_{h}$ into elements $K$ (polygons or polyhedra) and for every $K \in \mathcal{T}_{h}$, we consider the local space $V_{h}^{k}(K)$ with $k \geq 1$ as defined in (3.2). Then, the global nonconforming virtual element space $V_{h}^{k}$ of order $k$ is given by

$$
V_{h}^{k}=\left\{v_{h} \in H^{1, \mathrm{nc}}\left(\mathcal{T}_{h} ; k\right):\left.v_{h}\right|_{K} \in V_{h}^{k}(K) \quad \forall K \in \mathcal{T}_{h}\right\} .
$$

Arguing then as for a single element, we can compute the degrees of freedom of the global space:

$$
N_{\mathcal{T}_{h}}= \begin{cases}N_{\text {edges }} k+N_{\text {elements }}(k-1) k / 2 & \text { for } d=2, \\ N_{\text {faces }} k(k+1) / 2+N_{\text {elements }}(k-1) k(k+1) / 6 & \text { for } d=3,\end{cases}
$$

where $N_{\text {elements }}$ denotes the total number of elements $K$ of the partition $\mathcal{T}_{h}$ and $N_{\text {edges }}$ and $N_{\text {faces }}$ refer to the total number of edges (in $d=2$ ) and faces (in $d=3$ ), respectively; they are the cardinality of the set $\mathcal{E}_{h}$.

Arguing again as for a single element, as degrees of freedom for the global space $V_{h}^{k}$ we can take:

(i) the moments of $v_{h}$ of order up to $k-1$ on each $(d-1)$-dimensional edge/face $e \in \mathcal{E}_{h}$ :

$$
\mu_{e}^{k-1}\left(v_{h}\right)=\left\{\frac{1}{|e|} \int_{e} v_{h} m \mathrm{~d} s, \quad \forall m \in \mathcal{M}^{k-1}(e), \forall e \in \mathcal{E}_{h}\right\} ;
$$

(ii) the moments of $v_{h}$ of order up to $k-2$ on each $d$-dimensional element $K \in \mathcal{T}_{h}$ :

$$
\mu_{K}^{k-2}\left(v_{h}\right)=\left\{\frac{1}{|K|} \int_{K} v_{h} m \mathrm{~d} x, \quad \forall m \in \mathcal{M}^{k-2}(K), \forall K \in \mathcal{T}_{h}\right\} .
$$

As it happens for the local space $V_{h}^{k}(K)$, the dimension $N^{\text {tot }}$ given in (3.10) coincides with the total number of degrees of freedom (3.11) and (3.12). The unisolvence for the local space $V_{h}^{k}(K)$ given in Lemma 3.1, implies the unisolvence for the global space $V_{h}^{k}$. Since the proof is essentially the same, we omit it for conciseness. 


\subsection{Approximation properties}

Following [7], we now revise the local approximation properties by polynomial functions and functions in the virtual nonconforming space. In the former case, the approximation is the same as for the finite elements. In the latter case, the discussion is similar as for conforming VEM. We briefly recall both for completeness of exposition and future reference in the paper.

\section{Local approximation}

In view of the mesh regularity assumptions (A0), there exists a local polynomial approximation $w_{\pi} \in \mathbb{P}^{k}(K)$ for every smooth function $w$ defined on $K$. According to [12] for star-shaped elements and the generalization to the general shaped elements satisfying (A0) found in ([6], Sect. 1.6), the polynomial $w_{\pi}$ has optimal approximation properties. Thus, for every $w \in H^{s}(K)$ with $2 \leq s \leq k+1$ there exists a polynomial $w_{\pi}$ in $\mathbb{P}^{k}(K)$ such that

$$
\left\|w-w_{\pi}\right\|_{0, K}+h_{K}\left|w-w_{\pi}\right|_{1, K} \leq C h_{K}^{s}|w|_{s, K},
$$

where $C$ is a positive constant that only depends on the polynomial degree $k$ and the mesh regularity constant $\varrho$. The proof of the above approximation result can be done by proceeding as for classical finite elements (see, for instance, [42], which can be used taking into account (2.9)). Following always [42] one can trace how the constant $C$ depends on the constants appearing in hypothesis (A0) $\rho, \rho_{e}$ and the ratio between the maximum and minimum diameter of the star-shaped element. We refer to [42] for the explicit expressions.

\section{Interpolation error}

Following essentially $[2,7]$ we can define an interpolation operator in $V_{h}^{k}$ having optimal approximation properties. The idea is to use the degrees of freedom without requiring an explicit construction of the basis functions associated with them, since, unlike the finite element method, they are not needed for implementing or constructing the method. We assume that we have numbered the degrees of freedom (3.11) and (3.12) from $i=1, \ldots N_{\mathcal{T}_{h}}$, and that we have the canonical basis associated or induced by them (even if we do not compute such basis!). Let $\chi_{i}(\cdot)$ denote the operator that associates the $i$ th degree of freedom $\chi_{i}(v)$ with each smooth enough function $v$ :

$$
v \rightarrow \chi_{i}(v)=i \text { th degree of freedom of } v \quad \forall i=1, \ldots, N_{\mathcal{T}_{h}},
$$

and $\left\{\psi_{i}\right\}$ denote the set of the "canonical" shape functions of $V_{h}^{k}$ satisfying the condition $\chi_{i}\left(\psi_{j}\right)=\delta_{i j}$ for $i, j=1, \ldots, N_{\mathcal{T}_{h}}$. Then, from the previous construction of the space, it follows easily that for any $v \in H^{1, \text { nc }}\left(\mathcal{T}_{h} ; k\right)$ there exists a function $v^{I} \in V_{h}^{k}$ such that

$$
\chi_{i}\left(v-v^{I}\right)=0 \quad \forall i=1, \ldots N_{\mathcal{T}_{h}} .
$$

This is enough to guarantee that we can apply the classical results of approximation. In particular, there exists a constant $C>0$ independent of $h$ and such that for every $h>0$, every $K \in \mathcal{T}_{h}$, every $s$ with $2 \leq s \leq k+1$, and every $v \in H^{s}(K)$ the "interpolant" $v^{I} \in V_{h}^{k}$ given through (3.15) satisfies:

$$
\left\|v-v^{I}\right\|_{0, K}+h_{K}\left|v-v^{I}\right|_{1, K} \leq C h_{K}^{s}|v|_{s, K}
$$

The proof of the above approximation result can be done by proceeding as in ([35], Prop. 4.2) together with estimate (3.13) and classical results from finite elements. 


\subsection{Construction of the virtual bilinear form $a_{h}$}

We now tackle the second part of the definition of the nonconforming virtual discretization (3.1). The goal is to define a suitable symmetric bilinear form $a_{h}: V_{h}^{k} \times V_{h}^{k} \longrightarrow \mathbb{R}$ that enjoys good stability and approximation properties and is computable over all the functions in $V_{h}^{k}$. To this end, we first split $a_{h}(\cdot, \cdot)$ as we did for $a(\cdot, \cdot)$ in $(2.10)$ :

$$
a_{h}\left(u_{h}, v_{h}\right)=\sum_{K \in \mathcal{T}_{h}} a_{h}^{K}\left(u_{h}, v_{h}\right) \quad \forall u_{h}, v_{h} \in V_{h}^{k},
$$

with $a_{h}^{K}: V_{h}^{k}(K) \times V_{h}^{k}(K) \longrightarrow \mathbb{R}$ denoting the restriction of $a_{h}(\cdot, \cdot)$ to the local space $V_{h}^{k}(K)$. Then, we look at the local construction of $a_{h}^{K}(\cdot, \cdot)$.

We start by noticing that on each element $K$, for $p \in \mathbb{P}^{k}(K)$ and $v_{h} \in V_{h}^{k}(K)$ we can compute exactly $a^{K}\left(p, v_{h}\right)$ by using only the local degrees of freedom given in (3.5) and (3.6). In fact, since

$$
a^{K}\left(p, v_{h}\right)=\int_{K} \nabla p \cdot \nabla v_{h} \mathrm{~d} x=-\int_{K} v_{h} \Delta p \mathrm{~d} x+\int_{\partial K} v_{h} \frac{\partial p}{\partial \mathbf{n}} \mathrm{d} x
$$

we only needs to observe that the two integrals on the right hand side are determined exactly by the degrees of freedom (3.5) and (3.6), without requiring any further explicit knowledge of the function $v_{h}$ in $K$.

Now, to construct $a_{h}(\cdot, \cdot)$, always following [2], we first define a projection operator that can be thought of as the Ritz-Galerkin projection in finite elements. Let $\Pi_{K}^{\nabla}: H^{1}(K) \rightarrow \mathbb{P}^{k}(K)$ be defined by

$$
\int_{K} \nabla\left(\Pi_{K}^{\nabla}\left(v_{h}\right)-v_{h}\right) \cdot \nabla q \mathrm{~d} x=0 \quad \forall q \in \mathbb{P}^{k}(K), \forall v_{h} \in V_{h}^{k}(K)
$$

together with the condition

$$
\begin{array}{cc}
\int_{\partial K}\left(\Pi_{K}^{\nabla}\left(v_{h}\right)-v_{h}\right) \mathrm{d} s=0 & \text { if } k=1, \\
\int_{K}\left(\Pi_{K}^{\nabla}\left(v_{h}\right)-v_{h}\right) \mathrm{d} x=0 & \text { if } k \geq 2 .
\end{array}
$$

Note that $\Pi_{K}^{\nabla}(v)$ is indeed computable for any $v \in V_{h}^{k}$ from the degrees of freedom (3.5) and (3.6) in view of $(3.17)$ and the symmetry of the bilinear form. Also, $\Pi_{K}^{\nabla}$ is the identity operator on $\mathbb{P}^{k}(K)$, i.e., $\Pi_{K}^{\nabla}\left(\mathbb{P}^{k}(K)\right)=$ $\mathbb{P}^{k}(K)$.

We then define for every $u_{h}, v_{h} \in V_{h}^{k}(K)$ the bilinear form

$$
a_{h}^{K}\left(u_{h}, v_{h}\right)=a^{K}\left(\Pi_{K}^{\nabla}\left(u_{h}\right), \Pi_{K}^{\nabla}\left(v_{h}\right)\right)+S^{K}\left(u_{h}-\Pi_{K}^{\nabla}\left(u_{h}\right), v_{h}-\Pi_{K}^{\nabla}\left(v_{h}\right)\right),
$$

where the term $S^{K}(\cdot, \cdot)$ is a symmetric bilinear form whose matrix representation in the canonical basis functions $\left\{\psi_{i}\right\}$ of $V_{h}^{k}(K)$ is spectrally equivalent to the identity matrix scaled by the factor $\gamma_{K}$ defined as:

$$
\gamma_{K}=h^{d-2} .
$$

Thus, for every function $v_{h}$ in $V_{h}^{k}(K)$, it holds that

$$
S^{K}\left(v_{h}, v_{h}\right) \simeq h^{d-2} \boldsymbol{v}_{h}^{t} \boldsymbol{v}_{h}
$$

where $\boldsymbol{v}_{h}$ is the vector collecting the degrees of freedom of $v_{h}$. The scaling of $S^{K}$ guarantees that

$$
c_{*} a^{K}\left(v_{h}, v_{h}\right) \leq S^{K}\left(v_{h}, v_{h}\right) \leq c^{*} a^{K}\left(v_{h}, v_{h}\right) \quad \forall v_{h} \in \operatorname{ker}\left(\Pi_{K}^{\nabla}\right),
$$

for two positive constants $c_{*}$ and $c^{*}$ independent of $h$.

We now show that the construction of $a_{h}^{K}(\cdot, \cdot)$ guarantees the usual consistency and stability properties in VEM. 
Lemma 3.3. For all $h>0$ and for all $K \in \mathcal{T}_{h}$, the bilinear form $a_{h}^{K}(\cdot, \cdot)$ defined in (3.21) satisfies the following consistency and stability properties:

- $k$-Consistency:

$$
a_{h}^{K}\left(p, v_{h}\right)=a^{K}\left(p, v_{h}\right) \quad \forall p \in \mathbb{P}^{k}(K), \forall v_{h} \in V_{h}^{k}(K) .
$$

- Stability: there exists two positive constants $\alpha_{*}$ and $\alpha^{*}$ independent of mesh size $h$ but depending on the shape regularity of the partition such that

$$
\alpha_{*} a^{K}\left(v_{h}, v_{h}\right) \leq a_{h}^{K}\left(v_{h}, v_{h}\right) \leq \alpha^{*} a^{K}\left(v_{h}, v_{h}\right) \quad \forall v_{h} \in V_{h}^{k}(K) .
$$

Proof. The $k$-consistency property in (3.25) follows immediately from definition (3.21) and the fact that $\Pi_{K}^{\nabla}$ is the identity operator on $\mathbb{P}^{k}(K)$. Since $\Pi_{K}^{\nabla} \mathbb{P}^{k}(K)=\mathbb{P}^{k}(K)$, it follows that $S^{K}\left(p-\Pi_{K}^{\nabla}(p), v_{h}-\Pi_{K}^{\nabla}\left(v_{h}\right)\right)=0$ for every $p \in \mathbb{P}^{k}(K)$ and every $v_{h} \in V_{h}^{k}(K)$. So, using the definition of $\Pi_{K}^{\nabla}$ and the definition (3.21) we have

$$
a_{h}^{K}\left(p, v_{h}\right)=a^{K}\left(\Pi_{K}^{\nabla}(p), \Pi_{K}^{\nabla}\left(v_{h}\right)\right)=a^{K}\left(p, \Pi_{K}^{\nabla}\left(v_{h}\right)\right)=a^{K}\left(p, v_{h}\right),
$$

which gives (3.25) and proves the $k$-consistency property.

To show (3.26), from the definition of $a_{h}^{K}(\cdot, \cdot)$ given in (3.21), the symmetry of the bilinear form and (3.24), we have

$$
\begin{aligned}
a_{h}^{K}\left(v_{h}, v_{h}\right) & \leq a^{K}\left(\Pi_{K}^{\nabla}\left(v_{h}\right), \Pi_{K}^{\nabla}\left(v_{h}\right)\right)+c^{*} a^{K}\left(v_{h}-\Pi_{K}^{\nabla}\left(v_{h}\right), v_{h}-\Pi_{K}^{\nabla}\left(v_{h}\right)\right) \\
& \leq \max \left(1, c^{*}\right)\left(a^{K}\left(\Pi_{K}^{\nabla}\left(v_{h}\right), \Pi_{K}^{\nabla}\left(v_{h}\right)\right)+a^{K}\left(v_{h}-\Pi_{K}^{\nabla}\left(v_{h}\right), v_{h}-\Pi_{K}^{\nabla}\left(v_{h}\right)\right)\right) \\
& =\alpha^{*} a^{K}\left(v_{h}, v_{h}\right),
\end{aligned}
$$

and

$$
\begin{aligned}
a_{h}^{K}\left(v_{h}, v_{h}\right) & \geq a^{K}\left(\Pi_{K}^{\nabla}\left(v_{h}\right), \Pi_{K}^{\nabla}\left(v_{h}\right)\right)+c_{*} a^{K}\left(v_{h}-\Pi_{K}^{\nabla}\left(v_{h}\right), v_{h}-\Pi_{K}^{\nabla}\left(v_{h}\right)\right) \\
& \geq \min \left(1, c_{*}\right)\left(a^{K}\left(\Pi_{K}^{\nabla}\left(v_{h}\right), \Pi_{K}^{\nabla}\left(v_{h}\right)\right)+a^{K}\left(v_{h}-\Pi_{K}^{\nabla}\left(v_{h}\right), v_{h}-\Pi_{K}^{\nabla}\left(v_{h}\right)\right)\right) \\
& =\alpha_{*} a^{K}\left(v_{h}, v_{h}\right),
\end{aligned}
$$

which shows (3.26) with $\alpha_{*}=\min \left(1, c_{*}\right)$ and $\alpha^{*}=\max \left(1, c^{*}\right)$ and concludes the proof.

Cauchy-Schwarz inequality, together with (3.26) and the boundedness of the local continuous bilinear form give

$$
\begin{aligned}
a_{h}^{K}\left(u_{h}, v_{h}\right) & \leq\left(a_{h}^{K}\left(u_{h}, u_{h}\right)\right)^{1 / 2}\left(a_{h}^{K}\left(v_{h}, v_{h}\right)\right)^{1 / 2} \leq \alpha^{*}\left(a^{K}\left(u_{h}, u_{h}\right)\right)^{1 / 2}\left(a^{K}\left(v_{h}, v_{h}\right)\right)^{1 / 2} \\
& =\alpha^{*}\left\|\nabla u_{h}\right\|_{0, K}\left\|\nabla v_{h}\right\|_{0, K}
\end{aligned}
$$

which establishes the continuity of $a_{h}^{K}$.

\subsection{Construction of the right-hand side term $\left\langle\mathrm{f}_{\mathrm{h}}, \cdot\right\rangle$}

The forcing term is constructed in the same way as it is done for the conforming VEM. The idea is to use whenever possible the degrees of freedom (3.12) to compute $f_{h}$ exactly. Denoting by $\mathcal{P}_{K}^{\ell}: L^{2}(K) \longrightarrow \mathbb{P}^{\ell}(K)$ the $L^{2}$-orthogonal projection onto the space $\mathbb{P}^{\ell}(K)$ for $\ell \geq 0$, we define $f_{h}$ at the elemental level by:

$$
\left.f_{h}\right|_{K}=\left\{\begin{array}{ll}
\mathcal{P}_{K}^{0}(f) & \text { for } k=1, \\
\mathcal{P}_{K}^{k-2}(f) & \text { for } k \geq 2 .
\end{array} \quad \forall K \in \mathcal{T}_{h} .\right.
$$


In the definition above for $k \geq 2$, the right hand side $\left\langle f_{h}, v_{h}\right\rangle$ is fully computable for the functions in $V_{h}^{k}$ since:

$$
\left\langle f_{h}, v_{h}\right\rangle=\sum_{K \in \mathcal{T}_{h}} \int_{K} \mathcal{P}_{K}^{k-2}(f) v_{h} \mathrm{~d} x=\sum_{K \in \mathcal{T}_{h}} \int_{K} f \mathcal{P}_{K}^{k-2}\left(v_{h}\right) \mathrm{d} x,
$$

which is readily available from $(3.12)$.

For $k=1$ and each $K \in \mathcal{T}_{h}$ we first define:

$$
\left.\tilde{v}_{h}\right|_{K}=\frac{1}{n} \sum_{e \in \partial K} \frac{1}{|e|} \int_{e} v_{h} \mathrm{~d} s \approx \mathcal{P}_{K}^{0}\left(v_{h}\right)
$$

and notice that $\left.\tilde{v}_{h}\right|_{K}$ is a first-order approximation to $\mathcal{P}_{K}^{0}\left(v_{h}\right)=\frac{1}{|K|} \int_{K} v_{h} \mathrm{~d} x$, i.e., we have that $\left|\tilde{v}_{h}\right|_{K}-\mathcal{P}_{K}^{0}\left(v_{h}\right) \mid \leq$ $C h|v|_{1, K}$. Then, the idea is to use $\tilde{v}_{h}$ to compute the approximation to the right hand side of (3.1):

$$
\left\langle f_{h}, v_{h}\right\rangle=\sum_{K \in \mathcal{T}_{h}} \int_{K} \mathcal{P}_{K}^{0}(f) \tilde{v}_{h} \mathrm{~d} x \approx \sum_{K \in \mathcal{T}_{h}}|K| \mathcal{P}_{K}^{0}(f) \mathcal{P}_{K}^{0}\left(v_{h}\right) .
$$

Notice that the computation of the right-most term above would require the knowledge of the average value of $v_{h}$ on each element $K$ and such information is, in principle, not available. Therefore, we approximate $\mathcal{P}_{K}^{0}\left(v_{h}\right)$ by using the numerical quadrature rule defined by $\tilde{v}_{h}$ that only uses the moments $\mu_{e}^{0}\left(v_{h}\right)$ in (3.5).

Furthermore, in both cases $k \geq 2$ and $k=1$, an estimate for the error in the approximation is already available by using the definition of the $L^{2}$-projection, Cauchy-Schwarz and standard approximation estimates [18]. For $k \geq 2$ and $s \geq 1$ one easily has

$$
\begin{aligned}
\left|\left\langle f, v_{h}\right\rangle-\left\langle f_{h}, v_{h}\right\rangle\right| & =\left|\sum_{K \in \mathcal{T}_{h}} \int_{K}\left(f-\mathcal{P}_{K}^{k-2}(f)\right) v_{h} \mathrm{~d} x\right| \\
& =\left|\sum_{K \in \mathcal{T}_{h}} \int_{K}\left(f-\mathcal{P}_{K}^{k-2}(f)\right)\left(v_{h}-\mathcal{P}_{K}^{0}\left(v_{h}\right)\right) \mathrm{d} x\right| \\
& \leq\left\|f-\mathcal{P}_{K}^{k-2}(f)\right\|_{0, \Omega}\left\|v_{h}-\mathcal{P}_{K}^{0}\left(v_{h}\right)\right\|_{0, \Omega} \\
& \leq C h^{\min (k, s)}|f|_{s-1, \mathcal{T}_{h}}\left|v_{h}\right|_{1, h} .
\end{aligned}
$$

For $k=1$, the definition of $f_{h}$ together with using repeteadly the definition of the $L^{2}$-projection, Cauchy-Schwarz inequality and standard approximation estimates, give

$$
\begin{aligned}
\left|\left\langle f, v_{h}\right\rangle-\left\langle f_{h}, \tilde{v}_{h}\right\rangle\right| & =\left|\sum_{K \in \mathcal{T}_{h}} \int_{K}\left(f v_{h}-\mathcal{P}_{K}^{0}(f) \tilde{v}_{h}\right) \mathrm{d} x\right| \\
& \left.\leq \mid \sum_{K \in \mathcal{T}_{h}} \int_{K}\left(f-\mathcal{P}_{K}^{0}(f)\right) v_{h}\right) \mathrm{d} x|+| \sum_{K \in \mathcal{T}_{h}} \int_{K} \mathcal{P}_{K}^{0}(f)\left(v_{h}-\tilde{v}_{h}\right) \mathrm{d} x \mid \\
& \leq\left|\sum_{K \in \mathcal{T}_{h}} \int_{K}\left(f-\mathcal{P}_{K}^{0}(f)\right)\left(v_{h}-\mathcal{P}_{K}^{0}\left(v_{h}\right)\right) \mathrm{d} x\right|+\left\|\mathcal{P}_{K}^{0}(f)\right\|_{0, \Omega}\left\|v_{h}-\tilde{v}_{h}\right\|_{0, \Omega} \\
& \leq\left\|\mathcal{P}_{K}^{0}(f)-f\right\|_{0, \Omega}\left\|\mathcal{P}_{K}^{0}\left(v_{h}\right)-v_{h}\right\|_{0, \Omega}+C h\|f\|_{0, \Omega}\left|v_{h}\right|_{1, h} \\
& \leq C h\left(\|f\|_{0, \Omega}+|f|_{s-1, \mathcal{T}_{h}}\right)\left|v_{h}\right|_{1, h} .
\end{aligned}
$$

We finally mention that the estimate

$$
\left\|v_{h}-\tilde{v_{h}}\right\| \leq C h\|v\|_{1, h}
$$

was already indicated in [30]. We collect the results for $k=1$ and $k>1$ in the following lemma. 
Lemma 3.4. Let $k, s \geq 1, V_{h}^{k}$ the virtual element space defined by (3.9), $f \in H^{s-1}(\Omega), f_{h} \in\left(V_{h}^{k}\right)^{\prime}$ defined as in (3.28). For any $v_{h} \in V_{h}^{k}$ it holds:

$$
\left|\left\langle f, v_{h}\right\rangle-\left\langle f_{h}, v_{h}\right\rangle\right| \leq \begin{cases}C h\left(\|f\|_{0, \Omega}+|f|_{s-1, \mathcal{T}_{h}}\right)\left|v_{h}\right|_{1, h} & \text { for } k=1, \\ C h^{\min (k, s)}|f|_{s-1, \mathcal{T}_{h}}\left|v_{h}\right|_{1, h} & \text { for } k>1 .\end{cases}
$$

\subsection{Construction of the boundary term}

In the case of non-homogenous Dirichlet boundary conditions, we need to construct the corresponding boundary term. We define $g_{h}=\mathcal{P}_{e}^{k-1}(g)$ and observe that in view of the degrees of freedom (3.11), with such definition the boundary term will be fully computable. Indeed,

$$
\int_{\mathcal{E}_{h}^{\partial}} g_{h} v_{h} \mathrm{~d} s=\sum_{e \in \mathcal{E}_{h}^{\partial}} \int_{e} \mathcal{P}_{e}^{k-1}(g) v_{h} \mathrm{~d} s=\sum_{e \in \mathcal{E}_{h}^{\partial}} \int_{e} g \mathcal{P}_{e}^{k-1}\left(v_{h}\right) \mathrm{d} s \quad \forall v_{h} \in V_{h}^{k} .
$$

\section{ERROR ANALYSIS}

In this section we present the error analysis in the energy- and $L^{2}$-norm for the nonconforming virtual element approximation (3.1) to the model problem (2.3).

We start by noticing that the nonconformity of our discrete approximation space $V_{h}^{k} \subset H^{1, \text { nc }}\left(\mathcal{T}_{h} ; k\right) \nsubseteq H^{1}(\Omega)$ introduces a kind of consistency error in the approximation to the solution $u \in V$. In fact it should be noticed that using (2.10) together with standard integration by parts give

$$
\begin{aligned}
a(u, v) & =\sum_{K \in \mathcal{T}_{h}} \int_{K}-(\Delta u) v \mathrm{~d} x+\sum_{K \in \mathcal{T}_{h}} \int_{\partial K} \frac{\partial u}{\partial \mathbf{n}_{K}} v \mathrm{~d} s \\
& =(f, v)+\mathcal{N}_{h}(u, v) \quad \forall v \in H^{1, n c}\left(\mathcal{T}_{h} ; 1\right) .
\end{aligned}
$$

For $u \in H^{s}(\Omega), s \geq 3 / 2$, the term $\mathcal{N}_{h}$ can be rewritten using (2.7) as:

$$
\mathcal{N}_{h}(u, v)=\sum_{K \in \mathcal{T}_{h}} \int_{\partial K} \frac{\partial u}{\partial \mathbf{n}_{K}} v \mathrm{~d} s=\sum_{e \in \mathcal{E}_{h}} \int_{e} \nabla u \cdot \llbracket v \rrbracket \mathrm{d} s .
$$

The term $\mathcal{N}_{h}$ measures the extent to which the continuous solution $u$ fails to satisfy the virtual element formulation (3.1). In that respect, it could be regarded as a consistency error although it should be noted that such inconsistency here (as for the nonconforming finite element method) is due to the fact that the test functions $v_{h} \in V_{h} \nsubseteq V$, and therefore an error arises when using the variational formulation of the continuous solution (2.3).

We now provide an estimate for the term measuring the nonconformity. We have the following result

Lemma 4.1. Assume (A0) is satisfied. Let $k \geq 1$ and let $u \in H^{s+1}(\Omega)$ with $s \geq 1$ be the solution of (2.3). Let $v \in H^{1, n c}\left(\mathcal{T}_{h} ; 1\right)$ as defined in $(2.8)$. Then, there exists a constant $C>0$ depending only on the polynomial degree and the mesh regularity such that

$$
\left|\mathcal{N}_{h}(u, v)\right| \leq C h^{\min (s, k)}\|u\|_{s+1, \Omega}|v|_{1, h}
$$

where $\mathcal{N}_{h}(u, v)$ is defined in (4.2).

Proof. The proof follows along the same line as the one for the classical nonconforming methods. We briefly report it here for the sake of completeness. From the definition of the space $H^{1, n c}\left(\mathcal{T}_{h} ; k\right)$ with $k=1$, the definition 
of the $L^{2}(e)$-projection and Cauchy-Schwarz we find

$$
\begin{aligned}
\left|\mathcal{N}_{h}\left(u, v_{h}\right)\right| & =\left|\sum_{e \in \mathcal{E}_{h}} \int_{e}\left(\nabla u-\mathcal{P}_{e}^{k-1}(\nabla u)\right) \cdot \llbracket v_{h} \rrbracket \mathrm{d} s\right| \\
& =\left|\sum_{e \in \mathcal{E}_{h}} \int_{e}\left(\nabla u-\mathcal{P}_{e}^{k-1}(\nabla u)\right) \cdot\left(\llbracket v_{h} \rrbracket-\mathcal{P}_{e}^{0}\left(\llbracket v_{h} \rrbracket\right)\right) \mathrm{d} s\right| \\
& \leq \sum_{e \in \mathcal{E}_{h}}\left\|\nabla u-\mathcal{P}_{e}^{k-1}(\nabla u)\right\|_{0, e}\left\|\llbracket v_{h} \rrbracket-\mathcal{P}_{e}^{0}\left(\llbracket v_{h} \rrbracket\right)\right\|_{0, e},
\end{aligned}
$$

where $\mathcal{P}_{e}^{\ell}: L^{2}(e) \longrightarrow \mathbb{P}^{\ell}(e)$ is the $L^{2}$-orthogonal projection onto the space $\mathbb{P}^{\ell}(e)$ for $\ell \geq 0$.

Using now standard approximation estimates (see [18]) we have for each $e=\partial K^{+} \cap \partial K^{-}$,

$$
\begin{aligned}
& \left\|\nabla u-\mathcal{P}_{e}^{k-1}(\nabla u)\right\|_{0, e} \leq C h^{\min (s, k)-1 / 2}\|u\|_{s+1, K^{+} \cup K^{-}}, \\
& \left\|\llbracket v_{h} \rrbracket-\mathcal{P}_{e}^{0}\left(\llbracket v_{h} \rrbracket\right)\right\|_{0, e} \leq C h^{1 / 2}\left\|\nabla v_{h}\right\|_{0, K^{+} \cup K^{-}} .
\end{aligned}
$$

Hence, substituting the above estimates into (4.4) and summing over all elements, the proof is concluded.

Remark 4.2. To obtain at least an estimate of first order of the term $\mathcal{N}_{h}(u, v)$, notice that the proof of Lemma 4.1 requires further regularity (at least $u \in H^{2}(\Omega)$ ) than the one that problem (2.1) and (2.2) might have (as for instance in the case $f \in H^{-1}(\Omega)$ or even $f \in L^{2}(\Omega)$ and the domain not convex or with a second order problem with a jumping coefficient $\mathbb{K}$ ). We have followed the classical line for the error analysis to keep the presentation of the method simpler. Of course one might consider the extension of the results in [28] to estimate the nonconformity error arising in the nonconforming virtual approximation. We wish to note though, that such extension will require to have laid for virtual elements, some results on a-posteriori error estimation. While that would be surely possible and it might merit further investigation, it is out of the scope of this paper and we feel that by sticking to the present proof, we are able to convey in a better way (and with a neat presentation) the novelty and new idea of the paper.

We have the following result.

Theorem 4.3. Let (A0) be satisfied and let $u$ be the solution of (2.3). Consider the nonconforming virtual element method in (3.1), with $V_{h}^{k}$ given in (3.9) and with $a_{h}(\cdot, \cdot)$ and $f_{h} \in\left(V_{h}^{k}\right)^{\prime}$ defined as in Section 3. Then, problem (3.1) has a unique solution $u_{h} \in V_{h}^{k}$. Moreover, for every approximation $u^{I} \in V_{h}^{k}$ of $u$ and for every piecewise polynomial approximation $u_{\pi} \in \mathbb{P}^{k}\left(\mathcal{T}_{h}\right)$ of $u$, there exists a constant $C>0$ depending only on $\alpha_{*}$ and $\alpha^{*}$ in (3.26) such that the following estimate holds

$$
\left|u-u_{h}\right|_{1, h} \leq C\left(\left|u-u^{I}\right|_{1, h}+\left|u-u_{\pi}\right|_{1, h}+\sup _{v_{h} \in V_{h}^{k}} \frac{\left|\left\langle f-f_{h}, v_{h}\right\rangle\right|}{\left|v_{h}\right|_{1, h}}+\sup _{v_{h} \in V_{h}^{k}} \frac{\mathcal{N}_{h}\left(u, v_{h}\right)}{\left|v_{h}\right|_{1, h}}\right) .
$$

Furthermore, if $f \in H^{s-1}(\Omega)$ with $s \geq 1$, then we also have

$$
\left|u-u_{h}\right|_{1, h} \leq C h^{\min (k, s)}\left(\|u\|_{1+s, \Omega}+\|f\|_{s-1, \Omega}\right) .
$$

Proof. We first establish the existence and uniqueness of the solution to (3.1). From (3.27), (3.26) and (2.6) we easily have coercivity and continuity of the global discrete bilinear form in $H^{1, n c}\left(\mathcal{T}_{h} ; k\right)$ (and in particular in $\left.V_{h}^{k} \subset H^{1, n c}\left(\mathcal{T}_{h} ; k\right)\right)$

$$
\begin{array}{rlrl}
a_{h}(v, v) & \geq \alpha_{*} a(v, v) \geq C_{s} \alpha_{*}|v|_{1, h}^{2} & & \forall v \in H^{1, \mathrm{nc}}\left(\mathcal{T}_{h} ; k\right), \\
\left|a_{h}(u, v)\right| \leq \alpha^{*}|u|_{1, h}|v|_{1, h} & & \forall u, v \in H^{1, \mathrm{nc}}\left(\mathcal{T}_{h} ; k\right) .
\end{array}
$$


With $f_{h} \in\left(V_{h}^{k}\right)^{\prime}$ and the Poincaré inequality (2.9), a direct application of Lax-Milgram theorem guarantees existence and uniqueness of the solution $u_{h} \in V_{h}^{k}$ of (3.1).

We now prove the error estimate. We first write $u-u_{h}=\left(u-u^{I}\right)+\left(u^{I}-u_{h}\right)$ and use triangle inequality to bound

$$
\left|u-u_{h}\right|_{1, h} \leq\left|u-u^{I}\right|_{1, h}+\left|u_{h}-u^{I}\right|_{1, h} .
$$

The first term can be estimated using the standard approximation (3.16) and so it is enough to estimate the second term on the right hand side above. Let $\delta_{h}=u_{h}-u^{I} \in V_{h}^{k}$. Using the continuity (3.27) and the $k$-consistency several times

$$
\begin{aligned}
\alpha_{*}\left|\delta_{h}\right|_{1, h}^{2}= & \alpha_{*} a\left(\delta_{h}, \delta_{h}\right) \leq a_{h}\left(\delta_{h}, \delta_{h}\right) \\
= & a_{h}\left(u_{h}, \delta_{h}\right)-a_{h}\left(u^{I}, \delta_{h}\right) \\
= & \left(f_{h}, \delta_{h}\right)-\sum_{K \in \mathcal{T}_{h}} a_{h}^{K}\left(u^{I}-u_{\pi}, \delta_{h}\right)-\sum_{K \in \mathcal{T}_{h}} a_{h}^{K}\left(u_{\pi}, \delta_{h}\right) \\
= & \left(f_{h}, \delta_{h}\right)-\sum_{K \in \mathcal{T}_{h}} a_{h}^{K}\left(u^{I}-u_{\pi}, \delta_{h}\right)-\sum_{K \in \mathcal{T}_{h}} a^{K}\left(u_{\pi}, \delta_{h}\right) \\
= & \left(f_{h}, \delta_{h}\right)-\sum_{K \in \mathcal{T}_{h}} a_{h}^{K}\left(u^{I}-u_{\pi}, \delta_{h}\right)+\sum_{K \in \mathcal{T}_{h}} a^{K}\left(u-u_{\pi}, \delta_{h}\right)-a\left(u, \delta_{h}\right) \\
= & \left(f_{h}, \delta_{h}\right)-a\left(u, \delta_{h}\right)-\sum_{K \in \mathcal{T}_{h}} a_{h}^{K}\left(u^{I}-u_{\pi}, \delta_{h}\right)+\sum_{K \in \mathcal{T}_{h}} a^{K}\left(u-u_{\pi}, \delta_{h}\right) \\
= & \left(f_{h}, \delta_{h}\right)-\left(f, \delta_{h}\right)-\mathcal{N}_{h}\left(u, \delta_{h}\right)-\sum_{K \in \mathcal{T}_{h}} a_{h}^{K}\left(u^{I}-u_{\pi}, \delta_{h}\right) \\
& +\sum_{K \in \mathcal{T}_{h}} a^{K}\left(u-u_{\pi}, \delta_{h}\right)
\end{aligned}
$$

where in the last step we have used (4.1) to introduce the consistency error. The proof is then concluded by estimating each of the terms in the right hand side above and substituting in (4.8). Last part of the theorem, follows by using Lemmas 3.4 and 4.1 to bound the terms on the right hand side of (4.5).

Remark 4.4. Theorem 4.3 is the corresponding abstract result to ([7], Thm. 3.1). As commented before, the term $\mathcal{N}_{h}$ measures the extent to which the continuous solution $u$ fails to satisfy the virtual element formulation (3.1); measures the non-conformity of the approximation. In this respect, this result could be regarded as the analog for the VEM of the Strang Lemma for the finite element method.

\section{1. $L^{2}(\Omega)$-error analysis}

We now report the $L^{2}$ error analysis of the proposed nonconforming VEM. It follows closely the $L^{2}$-error analysis for classical nonconforming methods.

Theorem 4.5. Let $\Omega$ be a convex domain and let $\mathcal{T}_{h}$ be a family of partitions of $\Omega$ satisfying (A0). Let $k \geq 1$ and let $u \in H^{s+1}(\Omega), s \geq 1$ be the solution of (2.3) and let $u_{h} \in V_{h}^{k}$ be its nonconforming virtual element approximation solving (3.1). Then, there exists a positive constant $C$ depending on $k$, the regularity of the mesh and the shape of the domain such that

$$
\begin{aligned}
\left\|u-u_{h}\right\|_{0, \Omega} \leq & C h\left(\left|u-u_{h}\right|_{1, h}+\left|u-u_{\pi}\right|_{1, h}\right)+C\left(h^{2}+h^{\min (2, \bar{k}+1)}\right)\left\|f-f_{h}\right\|_{0, \Omega} \\
& +C h^{\min (k, s)+1}\|u\|_{s+1, \Omega} .
\end{aligned}
$$

where $\bar{k}=\max \{k-2,0\}$. 
Proof. We consider the dual problem: find $\psi \in H^{2}(\Omega) \cap H_{0}^{1}(\Omega)$ solution of

$$
-\Delta \psi=u-u_{h} \quad \text { in } \Omega, \quad \psi=0 \quad \text { on } \partial \Omega .
$$

From the assumptions on the domain, the elliptic regularity theory gives the inequality $\|\psi\|_{2, \Omega} \leq C\left\|u-u_{h}\right\|_{0, \Omega}$ where $C$ depends on the domain only through the domain's shape. Let $\psi^{I} \in V_{h}^{k}$ and $\psi_{\pi} \in \mathbb{P}^{k}\left(\mathcal{T}_{h}\right)$ be the approximations to $\psi$ satisfying (3.16) and (3.13).Then, integrating by parts we find

$$
\begin{aligned}
\left\|u-u_{h}\right\|_{0, \Omega}^{2} & =\int_{\Omega}-\Delta \psi\left(u-u_{h}\right) \mathrm{d} x \\
& =\sum_{K \in \mathcal{T}_{h}} \int_{K} \nabla \psi \cdot \nabla\left(u-u_{h}\right) \mathrm{d} x+\sum_{K \in \mathcal{T}_{h}} \int_{\partial K} \frac{\partial \psi}{\partial \mathbf{n}}\left(u-u_{h}\right) \mathrm{d} s \\
& =a\left(\psi-\psi^{I},\left(u-u_{h}\right)\right)+a\left(\psi^{I},\left(u-u_{h}\right)\right)+\mathcal{N}_{h}\left(\psi, u-u_{h}\right) .
\end{aligned}
$$

We now estimate the three terms above. The estimate for the first one follows from the continuity of $a(\cdot, \cdot)$ together with the approximation properties (3.16) of $\psi^{I}$ and the a priori estimate of $\psi$

$$
\left|a\left(\psi-\psi^{I}, u-u_{h}\right)\right| \leq C\left|\psi-\psi^{I}\right|_{1, h}\left|u-u_{h}\right|_{1, h} \leq C h\left\|u-u_{h}\right\|_{0, \Omega}\left|u-u_{h}\right|_{1, h} .
$$

Last term is readily estimated by means of Lemma 4.1 with $k=s=1$ (since obviously $u-u_{h} \in H^{1, n c}\left(\mathcal{T}_{h} ; 1\right)$ ), giving

$$
\left|\mathcal{N}_{h}\left(\psi, u-u_{h}\right)\right| \leq C h\|\psi\|_{2, \Omega}\left|u-u_{h}\right|_{1, h} \leq C h\left\|u-u_{h}\right\|_{0, \Omega}\left|u-u_{h}\right|_{1, h}
$$

To estimate the second term in (4.11) we use the symmetry of the problem together with (3.1) and (4.1) to write

$$
\begin{aligned}
a\left(\psi^{I}, u-u_{h}\right) & =a\left(u, \psi^{I}\right)-a\left(u_{h}, \psi^{I}\right) \\
& =\mathcal{N}_{h}\left(u, \psi^{I}\right)+\left\langle f, \psi_{i}\right\rangle-a\left(u_{h}, \psi^{I}\right)+a_{h}\left(u_{h}, \psi^{I}\right)-a_{h}\left(u_{h}, \psi^{I}\right) \\
& =\mathcal{N}_{h}\left(u, \psi^{I}\right)+\left\langle f-f_{h}, \psi^{I}\right\rangle+\left(a_{h}\left(u_{h}, \psi^{I}\right)-a\left(u_{h}, \psi^{I}\right)\right) \\
& =T_{0}+T_{1}+T_{2}
\end{aligned}
$$

To conclude we need to estimate each of the above terms. For the first one, we first notice that from the definition (4.2) and the regularity of $\psi$, one obviously has $\mathcal{N}_{h}(u, \psi)=0$. Hence, a standard application of Lemma 4.1 together with the approximation properties (3.16) of $\psi^{I}$ and the a priori estimate of $\psi$, gives

$$
\begin{aligned}
\left|T_{0}\right| & =\left|\mathcal{N}_{h}\left(u, \psi^{I}\right)\right|=\left|\mathcal{N}_{h}\left(u, \psi^{I}-\psi\right)\right| \leq C h^{\min (k, s)}\|u\|_{s+1, \Omega}\left|\psi^{I}-\psi\right|_{1, h} \\
& \leq C h^{\min (k, s)+1}\|u\|_{s+1, \Omega}\left\|u-u_{h}\right\|_{0, \Omega} .
\end{aligned}
$$

The last two terms in (4.12) can be bounded as in [8]. Here, we report the proof for the sake of completeness. For $T_{1}$, using the $L^{2}$-orthogonal projection, and denoting again $\bar{k}=\max \{k-2,0\}$, we find

$$
\begin{aligned}
& T_{1}=\sum_{K \in \mathcal{T}_{h}}\left(\int_{K}\left(f-f_{h}\right)\left(\psi^{I}-\psi\right) \mathrm{d} x+\int_{K}\left(f-f_{h}\right)\left(\psi-\mathcal{P}_{K}^{\bar{k}}(\psi)\right) \mathrm{d} x\right) \\
& \leq\left\|f-f_{h}\right\|_{0, \Omega}\left(\left\|\psi^{I}-\psi\right\|_{0, \Omega}+\left\|\psi-\mathcal{P}_{K}^{\bar{k}}(\psi)\right\|_{0, \Omega}\right) \\
& \leq C\left(h^{2}+h^{\min (2, \bar{k}+1)}\right)\left\|f-f_{h}\right\|_{0, \Omega}\left\|u-u_{h}\right\|_{0, \Omega} .
\end{aligned}
$$


As regards $T_{2}$, we use the symmetry together with the $k$-consistency property twice, and the definition of the norm $(2.6)$

$$
\begin{aligned}
T_{2} & =a_{h}\left(u_{h}, \psi^{I}\right)-a\left(u_{h}, \psi^{I}\right)=\sum_{K \in \mathcal{T}_{h}}\left(a_{h}^{K}\left(u_{h}-u_{\pi}, \psi^{I}\right)-a^{K}\left(u_{h}-u_{\pi}, \psi^{I}\right)\right) \\
& =\sum_{K \in \mathcal{T}_{h}}\left(a_{h}^{K}\left(u_{h}-u_{\pi}, \psi^{I}-\psi_{\pi}\right)-a^{K}\left(u_{h}-u_{\pi}, \psi^{I}-\psi_{\pi}\right)\right) \\
& \leq\left|u_{h}-u_{\pi}\right|_{1, h}\left|\psi_{h}-\psi_{\pi}\right|_{1, h} .
\end{aligned}
$$

Each of the above terms can be readily estimated by adding and subtracting $u$ and $\psi$ :

$$
\begin{aligned}
& \left|u_{h}-u_{\pi}\right|_{1, h}^{2} \leq \sum_{K \in \mathcal{T}_{h}}\left(\left\|\nabla\left(u_{h}-u\right)\right\|_{0, K}^{2}+\|\left.\nabla\left(u-u_{\pi}\right)\right|_{0, K} ^{2}\right) \\
& \left|\psi_{h}-\psi_{\pi}\right|_{1, h}^{2} \leq \sum_{K \in \mathcal{T}_{h}}\left(\left\|\nabla\left(\psi_{h}-\psi\right)\right\|_{0, K}^{2}+\|\left.\nabla\left(\psi-\psi_{\pi}\right)\right|_{0, K} ^{2}\right) \leq C h^{2}\left\|u-u_{h}\right\|_{0, \Omega}^{2},
\end{aligned}
$$

where in the last step we have also used the standard approximation properties (3.13) and (3.16). With the above estimates, the bound for the term $T_{2}$ finally reads

$$
T_{2} \leq C h\left\|u-u_{h}\right\|_{0, \Omega}\left(\left|u-u_{h}\right|_{1, h}+\left|u-u_{\pi}\right|_{1, h}\right)
$$

Plugging now the estimates for $T_{0}, T_{1}$ and $T_{2}$ into (4.12) we finally get:

$$
\begin{aligned}
\left\|u-u_{h}\right\|_{0, \Omega} \leq & C h\left(h^{\min (k, s)+1}\|u\|_{s+1, \Omega}+\left|u-u_{h}\right|_{1, h}+\left|u-u_{\pi}\right|_{1, h}\right)+C\left(h^{2}\right. \\
& \left.+h^{\min (2, \bar{k}+1)}\right)\left\|f-f_{h}\right\|_{0, \Omega},
\end{aligned}
$$

which concludes the proof.

\section{Connection with the nonconforming MFD method [30]}

In this section, we discuss the relationship between the proposed nonconforming VEM and the nonconforming MFD method in [30]. Throughout this section we will use the notation of [9]. Also, we will omit the element index $K$ from all the matrix symbols.

The stiffness matrix $\mathrm{M}^{\mathrm{VEM}}$ of the nonconforming VEM is formally defined as

$$
a_{h}^{K}\left(u_{h}, v_{h}\right)=\mathbf{v}_{h}^{T} \mathrm{M}^{\mathrm{VEM}} \mathbf{u}_{h},
$$

where $\mathbf{v}_{h}$ and $\mathbf{u}_{h}$ are algebraic vectors collecting the degrees of freedom of functions $v_{h}$ and $u_{h}$, respectively. We enumerate the whole set of $n_{K, k}$ scaled monomials used in (3.5) and (3.6) to define the degrees of freedom by local indices $i$ and $j$ (resp., $m_{i}$ and $m_{j}$ ) ranging from 1 to $n_{K, k}$.

To compute the stiffness matrix, we need two auxiliary matrices B and $\mathrm{D}$. The $j$ th column of matrix $\mathrm{B}$, for $j=1, \ldots, n_{K}$, is defined by

$$
\begin{aligned}
& \mathrm{B}_{1 j}= \begin{cases}\int_{\partial K} \psi_{j} \mathrm{~d} s=0 & \text { if } k=1, \\
\int_{K} \psi_{j} \mathrm{~d} x=0 & \text { if } k \geq 2,\end{cases} \\
& \mathrm{B}_{i j}=\int_{K} \nabla m_{i} \cdot \nabla \psi_{j} \mathrm{~d} x, \quad i=2, \ldots, n_{K, k} .
\end{aligned}
$$


The $j$ th column of matrix $\mathrm{D}$, for $j=1, \ldots, N_{K}$, collects the degrees of freedom of the $j$ th monomials and is defined by:

$$
\mathrm{D}_{i j}=\chi_{i}\left(m_{j}\right), \quad i=1, \ldots, n_{K} .
$$

Now, we consider the matrices $\mathrm{G}=\mathrm{BD}, \boldsymbol{\Pi}^{\nabla}=\mathrm{DG}^{-1} \mathrm{~B}$ and $\widetilde{\mathrm{G}}$, which is obtained from matrix $\mathrm{G}$ by setting its first row to zero. The VEM stiffness matrix is the sum of two matrices, $M^{V E M}=M_{0}^{V E M}+M_{1}^{V E M}$, which are defined by the following formula:

$$
\mathrm{M}^{\mathrm{VEM}}=\left(\mathrm{G}^{-1} \mathrm{~B}\right)^{T} \widetilde{\mathrm{G}}\left(\mathrm{G}^{-1} \mathrm{~B}\right)+\left(\mathrm{I}-\boldsymbol{\Pi}^{\nabla}\right)^{T} \mathrm{~S}\left(\mathrm{I}-\boldsymbol{\Pi}^{\nabla}\right),
$$

where I is the identity matrix and $\mathrm{S}$ is the matrix representation of the bilinear form $S^{K}$. The first matrix term corresponds to the consistency property and the second term ensures stability. According to (3.22), we can set

$$
\mathrm{S}=h^{d-2} \text { l. }
$$

Since the choice of $S^{K}$ is not unique, so is the choice of S; therefore, we have a family of virtual element schemes that differ by matrix $\mathrm{S}$.

The mimetic stiffness matrix considered in [30] has the same structure, $\mathrm{M}^{\mathrm{MFD}}=\mathrm{M}_{0}^{\mathrm{MFD}}+\mathrm{M}_{1}^{\mathrm{MFD}}$, and the two matrices $\mathrm{M}_{0}^{\mathrm{MFD}}$ and $\mathrm{M}_{1}^{\mathrm{MFD}}$ are also related to the consistency and stability properties. In particular, matrix $\mathrm{M}_{1}^{\mathrm{MFD}}$ is given by:

$$
\mathrm{M}_{1}^{\mathrm{MFD}}=\left(\mathrm{I}-\boldsymbol{\Pi}^{\perp}\right) \mathrm{U}\left(\mathrm{I}-\boldsymbol{\Pi}^{\perp}\right),
$$

where $\boldsymbol{\Pi}^{\perp}=\mathrm{D}\left(\mathrm{D}^{T} \mathrm{D}\right)^{-1} \mathrm{D}^{T}$ is the orthogonal projector on the linear space spanned by the columns of matrix $\mathrm{D}$ and $\mathrm{U}$ is a symmetric and positive definite matrix of parameters.

Since both the VEM and the MFD method use the same degrees of freedom, they must satisfy the same conditions of consistency and stability. Moreover, the matrices $\mathrm{M}_{0}^{\mathrm{MFD}}$ and $\mathrm{M}_{0}^{\mathrm{VEM}}$ are uniquely determined by the consistency condition (the exactness property on the same set of polynomials of degree $k$ ); thus, they must coincide. Consequently, the virtual and mimetic stiffness matrices may differ only for the stabilization terms $M_{1}^{\mathrm{VEM}}$ and $\mathrm{M}_{1}^{\mathrm{MFD}}$. The relation between $\mathrm{M}_{1}^{\mathrm{VEM}}$ and $\mathrm{M}_{1}^{\mathrm{MFD}}$ is established by the following lemma.

\section{Lemma 5.1.}

(i) For any mimetic stabilization matrix of the form (5.6), we can find a matrix $\mathrm{S}$ such that $\mathrm{M}_{1}^{\mathrm{VEM}}$ and $\mathrm{M}_{1}^{\mathrm{MFD}}$ coincide.

(ii) For any virtual element stabilization matrix as the second term in the right-hand-side of (5.4), we can find a matrix $\mathrm{U}$ such that $\mathrm{M}_{1}^{\mathrm{MFD}}$ and $\mathrm{M}_{1}^{\mathrm{VEM}}$ coincide.

Proof.

(i) A straightforward calculation shows that

$$
\boldsymbol{\Pi}^{\nabla} \boldsymbol{\Pi}^{\perp}=\boldsymbol{\Pi}^{\perp}, \quad\left(\boldsymbol{\Pi}^{\nabla}\right)^{T} \boldsymbol{\Pi}^{\perp}=\left(\boldsymbol{\Pi}^{\nabla}\right)^{T}, \quad \boldsymbol{\Pi}^{\perp} \boldsymbol{\Pi}^{\nabla}=\boldsymbol{\Pi}^{\nabla} .
$$

We take $\mathrm{S}=\mathrm{M}_{1}^{\mathrm{MFD}}$. Using (5.7) yields:

$$
\mathrm{M}_{1}^{\mathrm{VEM}}=\left(\mathrm{I}-\boldsymbol{\Pi}^{\nabla}\right)^{T}\left(\mathrm{I}-\boldsymbol{\Pi}^{\perp}\right) \mathrm{U}\left(\mathrm{I}-\boldsymbol{\Pi}^{\perp}\right)\left(\mathrm{I}-\boldsymbol{\Pi}^{\nabla}\right)=\mathrm{M}_{1}^{\mathrm{MFD}} .
$$

(ii) The relations in (5.7) imply that $\left(\mathbf{I}-\boldsymbol{\Pi}^{\perp}\right)\left(\mathrm{I}-\boldsymbol{\Pi}^{\nabla}\right)^{T}=\left(\mathrm{I}-\boldsymbol{\Pi}^{\nabla}\right)^{T}$. The assertion of the lemma follows by taking

$$
\mathrm{U}=\left(\mathrm{I}-\boldsymbol{\Pi}^{\nabla}\right)^{T} \mathrm{~S}\left(\mathrm{I}-\boldsymbol{\Pi}^{\nabla}\right)=\mathrm{M}_{1}^{\mathrm{VEM}} .
$$

This proves the assertion of the lemma.

Remark 5.2. An effective and practical choice in the mimetic technology (see [30]) is provided by taking $\mathbf{U}=\rho \mathbf{l}$ where $\rho$ is a scaling factor defined as the mean trace of $\mathrm{M}_{0}^{\mathrm{MFD}}$. This implies that $\mathrm{M}_{1}^{\mathrm{MFD}}=\rho\left(\mathrm{I}-\boldsymbol{\Pi}^{\perp}\right)$. 


\section{Numerical EXPERIMENTS}

The numerical experiments presented in this section are devoted to verify the theory presented in the previous sections.

To study the accuracy of the method we solve the diffusion equation on the domain $\Omega=] 0,1[\times] 0,1[$ using both constant and variable diffusion coefficients. For the constant coefficient case we consider problem (2.1)(2.2), while for the variable coefficient case, the Laplace operator " $\Delta u$ " in equation (2.1) is substituted by the more general elliptic operator " $\operatorname{div}(\Lambda \nabla u)$ ", where $\Lambda$ is the full diffusion tensor given by

$$
\Lambda(x, y)=\left(\begin{array}{cc}
1+y^{2} & -x y \\
-x y & 1+x^{2}
\end{array}\right) .
$$

The forcing term $f$ in (2.1) and the boundary function $g$ in (2.2) are set so that the exact solution of the problem is given by:

$$
u(x, y)=\sin (2 \pi x) \sin (2 \pi y)+x^{6}+y^{6} .
$$

We consider four different sequences of five meshes, labeled by $\left\{\mathcal{T}_{h}\right\}_{h}^{(1)},\left\{\mathcal{T}_{h}\right\}_{h}^{(2)},\left\{\mathcal{T}_{h}\right\}_{h}^{(3)}$, and $\left\{\mathcal{T}_{h}\right\}_{h}^{(4)}$, respectively. Figures $6 \mathrm{a}-6 \mathrm{~d}$ show the first and second mesh of each sequence (left and right plot, respectively). The meshes in $\left\{\mathcal{T}_{h}\right\}_{h}^{(1)}$ are built as follows. First, we determine a primal mesh by remapping the position $(\widehat{x}, \widehat{y})$ of the nodes of a uniform square partition of $\Omega$ by the smooth coordinate transformation (see [14]):

$$
\begin{aligned}
& x=\widehat{x}+0.1 \sin (2 \pi \widehat{x}) \sin (2 \pi \widehat{y}), \\
& y=\widehat{y}+0.1 \sin (2 \pi \widehat{x}) \sin (2 \pi \widehat{y}) .
\end{aligned}
$$

The corresponding mesh of $\left\{\mathcal{T}_{h}\right\}_{h}^{(1)}$ is built from the primal mesh by splitting each quadrilateral cell into two triangles and connecting the barycenters of adjacent triangular cells by a straight segment. The mesh construction is completed at the boundary by connecting the barycenters of the triangular cells close to the boundary to the midpoints of the boundary edges and these latters to the boundary vertices of the primal mesh. The meshes in $\left\{\mathcal{T}_{h}\right\}_{h}^{(2)}$ are built by partitioning the domain $\Omega$ into square cells and relocating each interior node to a random position inside a square box centered at that node. The sides of this square box are aligned with the coordinate axis and their length is equal to 0.8 times the minimum distance between two adjacent nodes of the initial square mesh. The meshes in $\left\{\mathcal{T}_{h}\right\}_{h}^{(3)}$ are obtained by filling the unit square with a suitably scaled non-convex octagonal cell, which is cut at the domain boundaries to fit into the unit square domain. The meshes in $\left\{\mathcal{T}_{h}\right\}_{h}^{(4)}$ are obtained by partitioning $\Omega$ by squares, each one divided into two triangles. All the meshes are parametrized by the number of partitions in each direction. The starting mesh of every sequence is built from a $5 \times 5$ regular grid, and the refined meshes are obtained by doubling this resolution.

For $k \geq 1$, we measure the relative errors

$$
E_{L^{2}}=\frac{\left(\sum_{K \in \mathcal{T}_{h}}\left\|u-\Pi_{K}^{\nabla} u_{h}\right\|_{0, K}^{2}\right)^{1 / 2}}{\|u\|_{0, \Omega}}
$$

and

$$
E_{H^{1}}=\frac{\left(\sum_{K \in \mathcal{T}_{h}}\left|u-\Pi_{K}^{\nabla} u_{h}\right|_{1, K}^{2}\right)^{1 / 2}}{|u|_{1, \Omega}} .
$$

Thus, on every element $K \in \mathcal{T}_{h}$, error $E_{L^{2}}$ compares the exact solution $u$ of problem (2.1) and (2.2), and $\Pi_{K}^{\nabla} u_{h}$, the projection of the virtual element solution $u_{h}$ of the discrete problem (3.1) onto $\mathbb{P}^{k}(K)$. Similarly, on every 

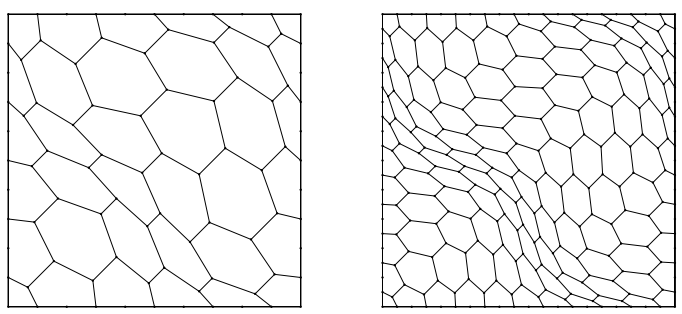

(a) Meshes of smoothly remapped hexagons $\left\{\mathcal{T}_{h}\right\}_{h}^{(1)}$
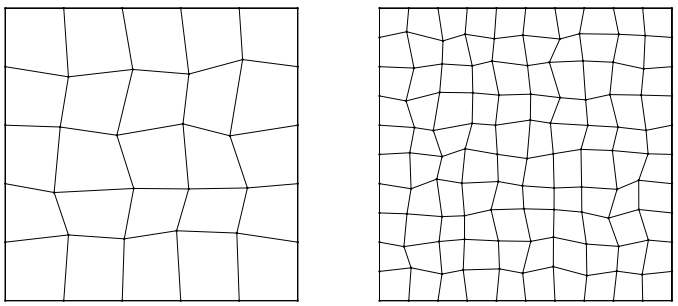

(b) Meshes of randomized quadrilaterals $\left\{\mathcal{T}_{h}\right\}_{h}^{(2)}$
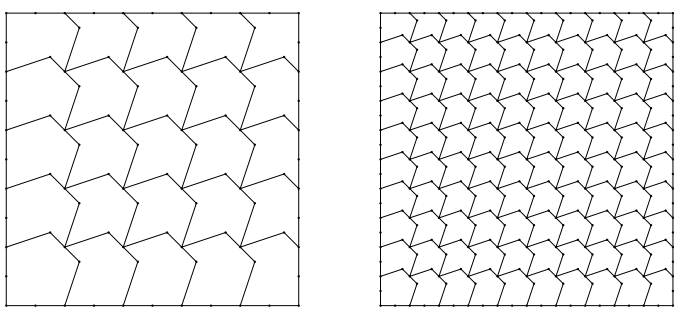

(c) Meshes of regular non-convex octagons $\left\{\mathcal{T}_{h}\right\}_{h}^{(3)}$
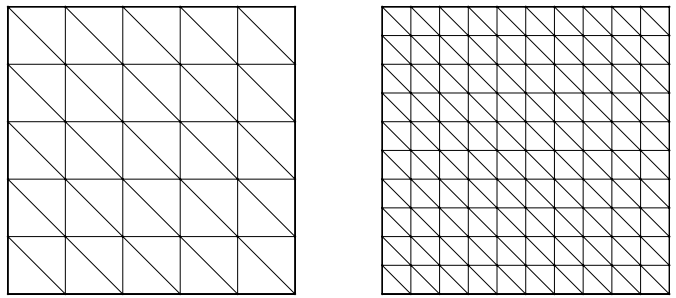

(d) Meshes of regular triangular cells $\left\{\mathcal{T}_{h}\right\}_{h}^{(4)}$

Figure 6 . The first two meshes of each mesh sequence that are used in the convergence and comparison tests of the numerical experiment section.

element $K \in \mathcal{T}_{h}$, error $E_{H^{1}}$ compares $\nabla u$ and $\nabla \Pi_{K}^{\nabla} u_{h}$. These relative errors are used to depict the convergence diagrams (error versus mesh size parameter $h$ ) in Figures 7-11. In Figure 12 is depicted the convergence diagram of the error versus the number of degrees of freedom. In each figure, the $E_{L^{2}}$ convergence diagram is given on the left, and on the right the corresponding $E_{H^{1}}$-convergence diagram. From the a priori analysis of Section 4, errors $E_{L^{2}}$ and $E_{H^{1}}$ must decrease proportionally to $h^{k+1}$ and $h^{k}$, respectively, when we apply the VEM of order $k$, i.e., the VEM using the virtual element space $V_{k}^{h}$. Accordingly, the experimental slopes for $E_{L^{2}}$ and $E_{H^{1}}$ must 

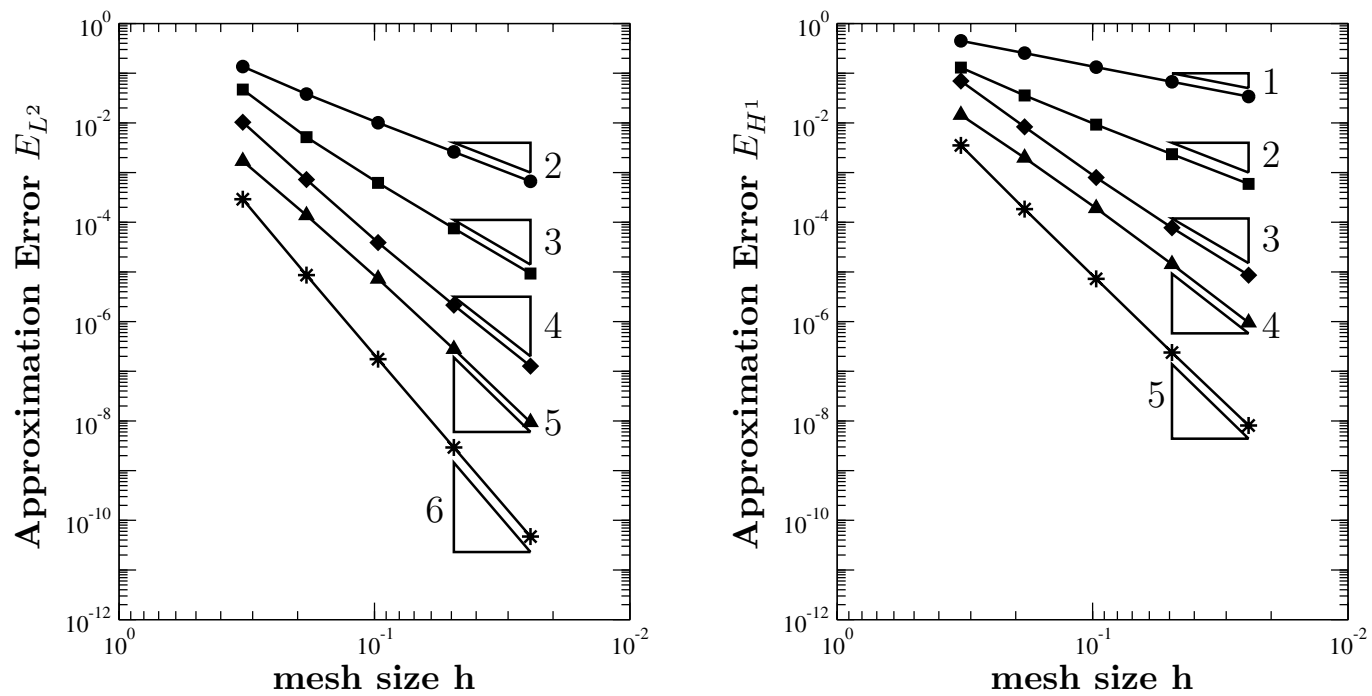

FiguRE 7. Relative error curves with respect to the mesh size $h$ for the numerical solution of the Poisson problem with constant diffusion coefficients on a sequence of meshes of smoothly remapped hexagons (see Fig. 6a). The VEM is based on the polynomials of degree $k=1$ (circles), $k=2$ (squares), $k=3$ (diamonds), $k=4$ (triangles), $k=5$ (stars).
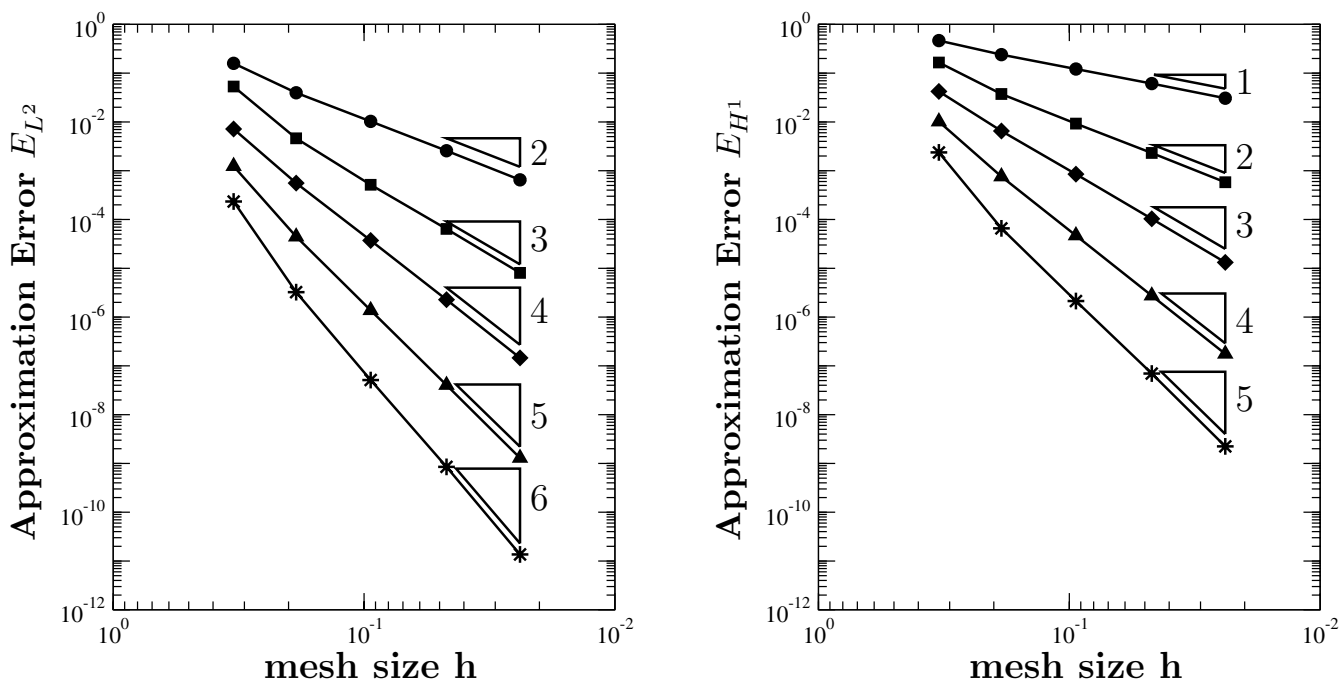

Figure 8. Relative error curves with respect to the mesh size $h$ for the numerical solution of the Poisson problem with constant diffusion coefficients on a sequence of meshes of randomized quadrilateral cells (see Fig. 6b). The VEM is based on the polynomials of degree $k=1$ (circles), $k=2$ (squares), $k=3$ (diamonds), $k=4$ (triangles), $k=5$ (stars).

be closed to $k+1$ and $k$ when we plot the error curves versus the mesh size parameter $h$. Instead, these slopes must be closed to $(k+1) / 2$ and $k / 2$ when we plot the error curves versus the number of degrees of freedom (as is done in Fig. 12) because this latter is roughly proportional to $h^{-1 / 2}$. As can be observed from the graphics, 

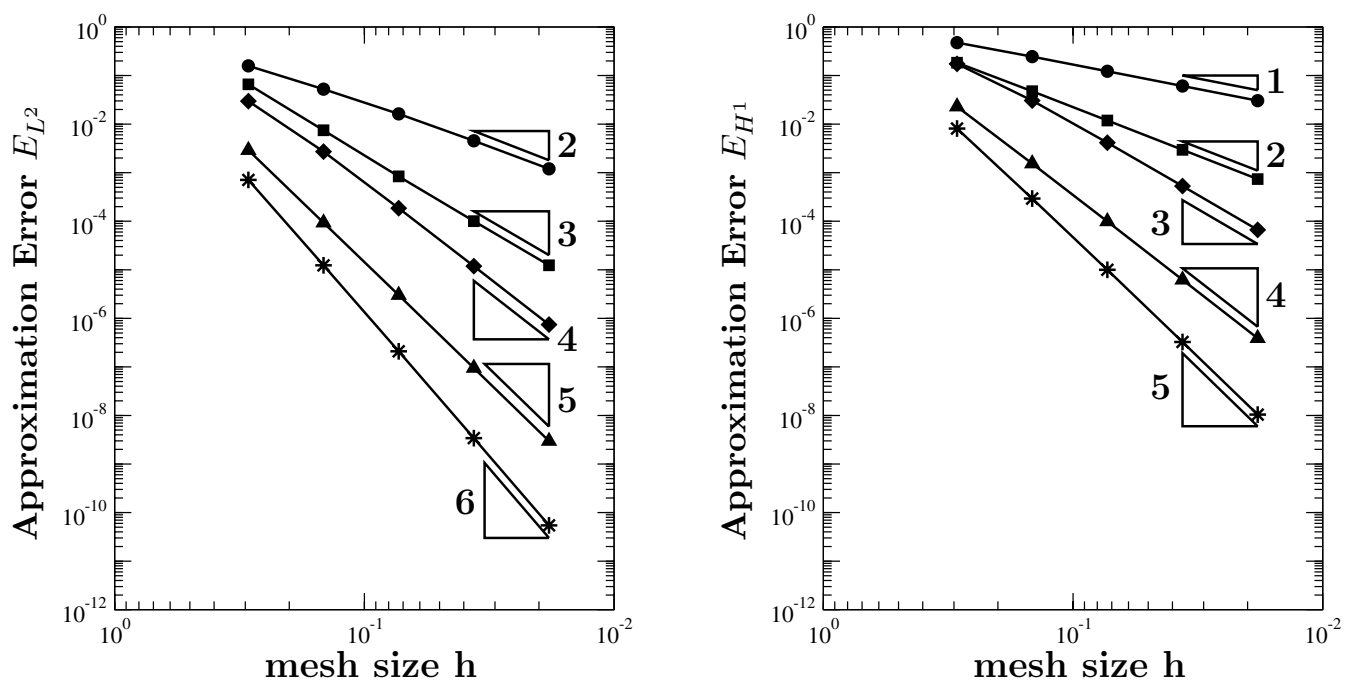

Figure 9. Relative error curves with respect to the mesh size $h$ for the numerical solution of the Poisson problem with constant diffusion coefficients on a sequence of meshes of regular non-convex octagons (see Fig. 6c). The VEM is based on the polynomials of degree $k=1$ (circles), $k=2$ (squares), $k=3$ (diamonds), $k=4$ (triangles), $k=5$ (stars).
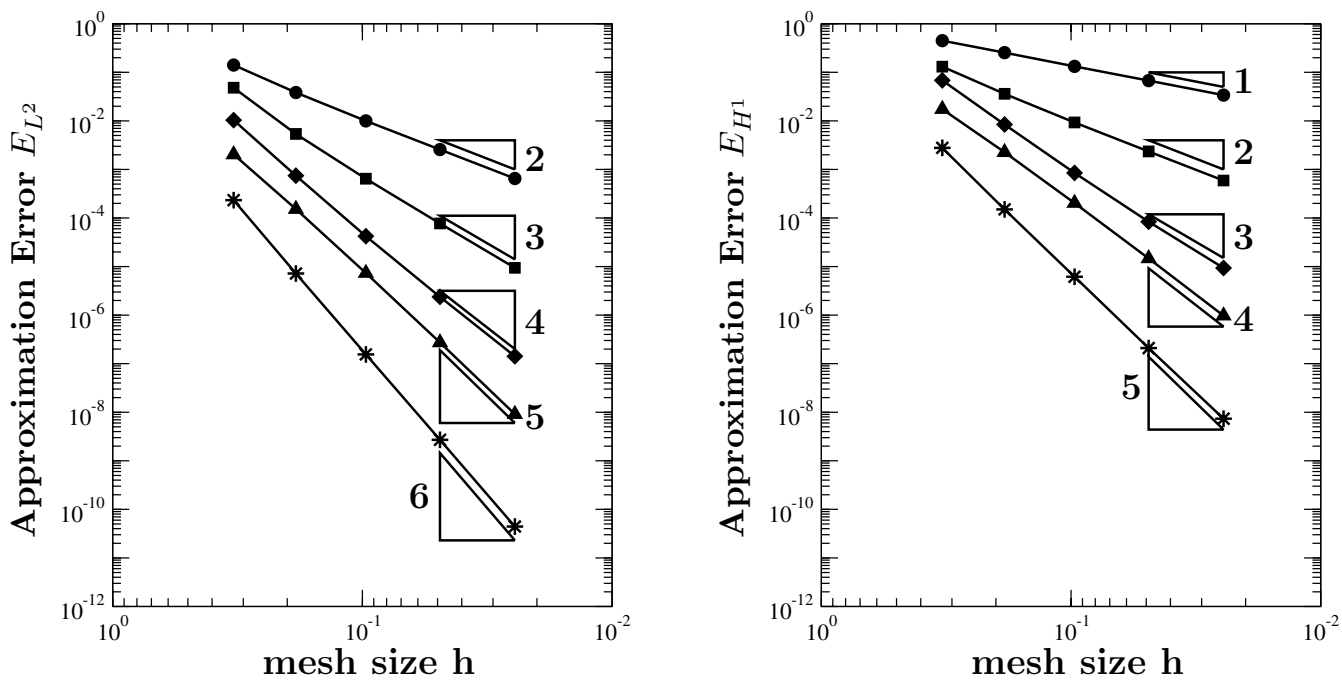

Figure 10. Relative error curves with respect to the mesh size $h$ for the numerical solution of the Poisson problem with variable diffusion coefficients on a sequence of meshes of smoothy remapped hexagons (see Fig. 6a). The VEM is based on the polynomials of degree $k=1$ (circles), $k=2$ (squares), $k=3$ (diamonds), $k=4$ (triangles), $k=5$ (stars).

for the different values of the polynomial degree $k$, the method converges as predicted by the theory. To ease the verification, the theoretical convergence rates are indicated by a number shown near the triangle depicted for each curve.

Figures 7, 8, and 9 show the error curves when we solve (2.1) and (2.2), i.e., the Poisson problem with constant coefficients, on the mesh families $\left\{\mathcal{T}_{h}\right\}_{h}^{(1)},\left\{\mathcal{T}_{h}\right\}_{h}^{(2)}$ and $\left\{\mathcal{T}_{h}\right\}_{h}^{(3)}$, respectively. Figures 10 shows the error 

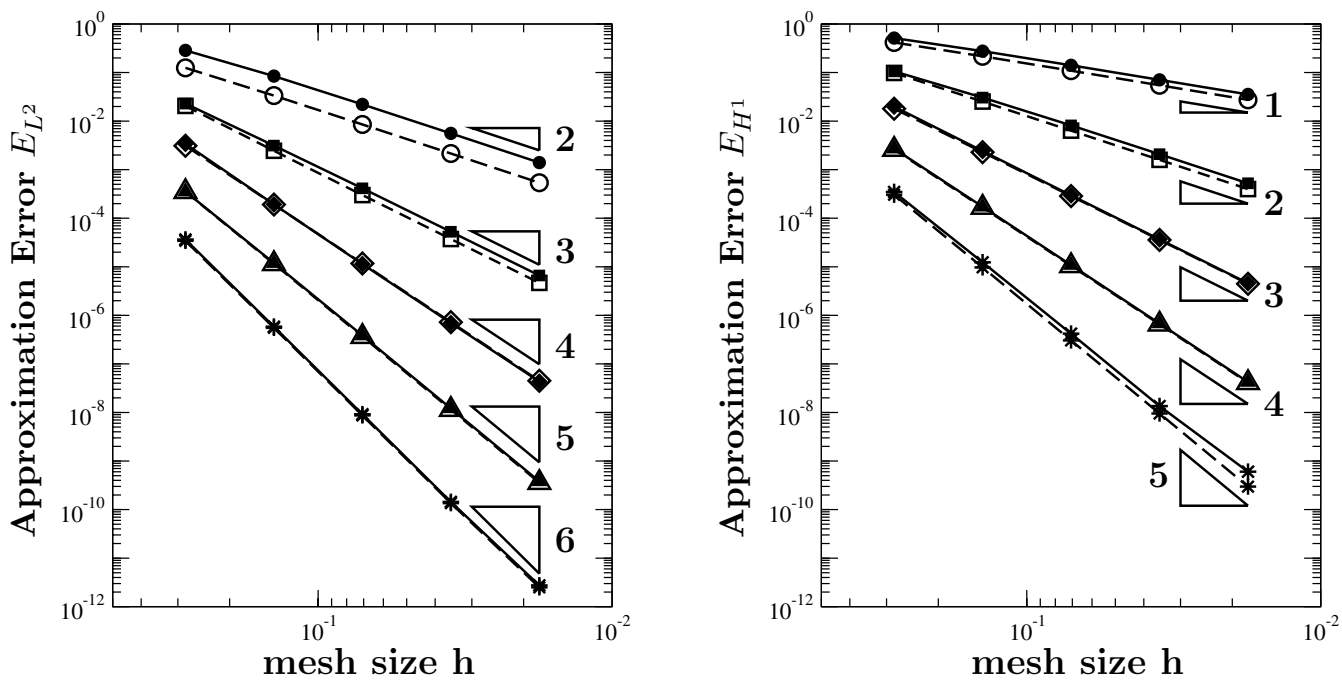

FIGURE 11. Relative error curves for VEM (solid) and non-conforming FEM (dashed) with respect to the mesh size $h$ for the numerical solution of the Poisson problem with constant diffusion coefficients on a sequence of regular meshes of triangles (see Fig. 6d). The schemes use polynomials of degree $k=1$ (circles), $k=2$ (squares), $k=3$ (diamonds), $k=4$ (triangles), $k=5$ (stars).
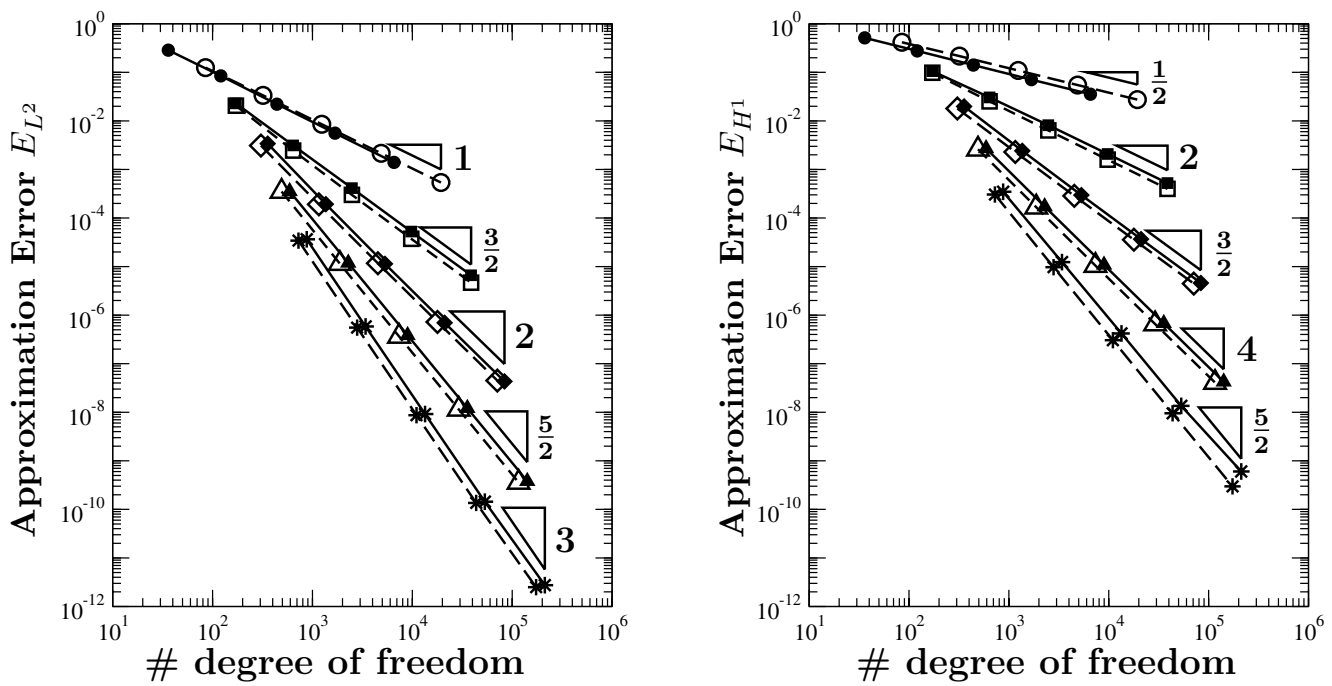

FIgURE 12. Relative error curves for VEM (solid) and non-conforming FEM (dashed) with respect to the number of degrees of freedom for the numerical solution of the Poisson problem with constant diffusion coefficients on a sequence of regular meshes of triangles (see Fig. 6d). The schemes use polynomials of degree $k=1$ (circles), $k=2$ (squares), $k=3$ (diamonds), $k=4$ (triangles), $k=5$ (stars).

curves when we solve the Poisson problem with the variable diffusion tensor $\Lambda$ using mesh family $\left\{\mathcal{T}_{h}\right\}_{h}^{(1)}$. The experimental convergence rates are in good agreement with the theoretical ones for all such calculations. 
Figures 11 and 12 compare the accuracy of the non-conforming VEM with different non-conforming finite element implementations with respect to $h$ and the number of degrees of freedom (notice the fractional convergence rates in this latter figure for the reason that we explained above). For comparison's sake, we solved problem (2.1) and (2.2) on the sequence of triangular meshes $\left\{\mathcal{T}_{h}\right\}_{h}^{(4)}$. The errors curves of the non-conforming VEM are displayed by solid lines, while those of the non-conforming FEM by dashed lines. For the comparison test, we implemented the Crouziex-Raviart FEM [20] for $k=1$, the Fortin-Soulie FEM [27] for $k=2$, the Crouzeix-Falk [21] for $k=3$, and the non-conforming finite element methods introduced by Baran-Stoyan [4] for $k=4$ and 5 . As in almost all the cases the error curves for the non-conforming VEM and FEM are indistinguishable, we conclude that the performance of the non-conforming VEM is almost always comparable to the performance of the non-conforming FEM that has the same order of accuracy. The only case where the performance of the non-conforming VEM seems to be inferior to that of the corresponding FEM is for $k=1$. Notice that in such a case, the VEM is penalized by the fact that it requires one more internal degrees of freedom in each triangle as is shown in Figure 1.

Finally, it is worth mentioning that in a preliminary stage of this work, the consistency of the nonconforming VEM of order $k$, i.e., the exactness of the method for polynomial solutions of degree $k$, has been tested numerically by solving problem (2.1) and (2.2) with boundary and source data determined by $u(x, y)=x^{k}+y^{k}$. To this purpose, we considered a wider set of polygonal meshes (including the four considered above) and values of $k$ from 1 to 5 . In all the cases, the magnitude of the errors $\mathcal{E}_{L^{2}}^{k}$ and $\mathcal{E}_{H^{1}}^{k}$ was comparable to the arithmetic precision, thus confirming the $k$-consistency property stated in (3.25). These results are not reported here.

\section{Conclusions}

In this work, we introduced the non-conforming virtual element method (VEM) for an elliptic equation. The VEM allows us to built arbitrary order schemes on shape-regular polygonal and polyhedral meshes that may include non-convex and degenerate elements. In contrast to the non-conforming finite element method, the construction of the VEM is done at once for any degree $k \geq 1$ and any element shape. Another advantage of the virtual element framework is ability to carry out theoretical analysis for complex meshes reusing many existing functional analysis tools. We have shown the optimal convergence estimates in the energy and $L^{2}$ norms. We also established an algebraic equivalence of the VEM and the mimetic finite difference method from [30]. Numerous numerical results presented here verify our theoretical conclusions and show that the new method is competitive with "classical" nonconforming finite element schemes with the same accuracy.

Acknowledgements. The first author is in-debt with Proff. F. Brezzi and D. Marini from Pavia, for the multiple and fruitful discussions and specially for the encouragement to carry out this work. The work of the first author was partially supported by KAUST grants BAS/1/1636-01-01 and Pocket ID 1000000193. She thanks KAUST for the support and hospitality, where part of the work was completed while she was Research Scientist with Peter Markowich. The work of the second and third authors was partially supported by the Laboratory Directed Research and Development Program (LDRD), U.S. Department of Energy Office of Science, Office of Fusion Energy Sciences, and the DOE Office of Science Advanced Scientific Computing Research (ASCR) Program in Applied Mathematics Research, under the auspices of the National Nuclear Security Administration of the U.S. Department of Energy by Los Alamos National Laboratory, operated by Los Alamos National Security LLC under Contract DE-AC52-06NA25396.

\section{REFERENCES}

[1] R.A. Adams, Sobolev spaces, Vol. 65 of Pure and Applied Mathematics. Academic Press [A subsidiary of Harcourt Brace Jovanovich, Publishers], New York-London (1975).

[2] B. Ahmad, A. Alsaedi, F. Brezzi, L.D. Marini and A. Russo, Equivalent projectors for virtual element methods. Comput. Math. Appl. 66 (2013) 376-391.

[3] D.N. Arnold and F. Brezzi, Mixed and nonconforming finite element methods: implementation, postprocessing and error estimates. RAIRO M2AN 19 (1985) 7-32.

[4] A.E. Baran and G. Stoyan, Gauss-Legendre elements: A stable, higher order non-conforming finite element family. Comput. 79 (2007) 1-21. 
[5] L. Beirão da Veiga, K. Lipnikov and G. Manzini, Arbitrary-order nodal mimetic discretizations of elliptic problems on polygonal meshes. SIAM J. Numer. Anal. 49 (2011) 1737-1760.

[6] L. Beirão da Veiga, K. Lipnikov and G. Manzini, The Mimetic Finite Difference Method. Vol. 11 of Modeling, Simulations and Applications, 1st edition. Springer-Verlag, New York (2014).

[7] L. Beirão da Veiga, F. Brezzi, A. Cangiani, G. Manzini, L.D. Marini and A. Russo, Basic principles of virtual element methods. Math. Models Methods Appl. Sci. 23 (2013) 199-214.

[8] L. Beirão da Veiga, F. Brezzi and L.D. Marini, Virtual elements for linear elasticity problems. SIAM J. Numer. Anal. 51 (2013) 794-812.

[9] L. Beirão da Veiga, F. Brezzi, L.D. Marini and A. Russo, The hitchhiker's guide to the virtual element method. Math. Models Methods Appl Sci. 24 (2014) 1541-1573.

[10] F.M. Benedetto, S. Berrone, S. Pieraccini and S. Scialò, The virtual element method for discrete fracture network simulations. Comput. Methods Appl. Mech. Engrg. 280 (2014) 135-156.

[11] S.C. Brenner, Poincaré-Friedrichs inequalities for piecewise $H^{1}$ functions. SIAM J. Numer. Anal. 41 (2003) 306-324.

[12] S.C. Brenner and L.R. Scott, The mathematical theory of finite element methods. Vol. 15 of Texts Appl. Math. Springer-Verlag, New York (1994).

[13] F. Brezzi and L.D. Marini, Virtual element methods for plate bending problems. Comput. Methods Appl. Mech. Engrg. 253 (2013) 455-462.

[14] F. Brezzi, K. Lipnikov and M. Shashkov, Convergence of the mimetic finite difference method for diffusion problems on polyhedral meshes. SIAM J. Numer. Anal. 43 (2005) 1872-1896.

[15] F. Brezzi, A. Buffa and K. Lipnikov, Mimetic finite differences for elliptic problems. ESAIM: M2AN 43 (2009) $277-295$.

[16] F. Brezzi, R.S. Falk and L.D. Marini, Basic principles of mixed virtual element methods. ESAIM: M2AN 48 (2014) $1227-1240$.

[17] A. Cangiani, G. Manzini and A. Russo, Convergence analysis of the mimetic finite difference method for elliptic problems. SIAM J. Numer. Anal. 47 (2009) 2612-2637.

[18] P.G. Ciarlet, Basic Error Estimates for Elliptic Problems. In Vol. II of Handb. Numer. Anal. North-Holland, Amsterdam (1991) $17-351$.

[19] M.I. Comodi, The Hellan-Herrmann-Johnson method: some new error estimates and postprocessing. Math. Comput. 52 (1989) $17-29$.

[20] M. Crouzeix and P.-A. Raviart, Conforming and nonconforming finite element methods for solving the stationary Stokes equations. I. Rev. Française Automat. Informat. Recherche Opérationnelle Sér. Rouge 7 (1973) 33-75.

[21] M. Crouzeix and R.S. Falk, Nonconforming finite elements for the Stokes problem. Math. Comput. 52 (1989) $437-456$.

[22] D.A. Di Pietro and A. Ern, A hybrid high-order locking-free method for linear elasticity on general meshes. Comput. Methods Appl. Mech. Engrg. 283 (2015) 1-21.

[23] D.A. Di Pietro and S. Lemaire, An extension of the Crouzeix-Raviart space to general meshes with application to quasiincompressible linear elasticity and Stokes flow. Math. Comput. 84 (2015) 1-31.

[24] D.A. Di Pietro, A. Ern and S. Lemaire, An arbitrary-order and compact-stencil discretization of diffusion on general meshes based on local reconstruction operators. Comput. Methods Appl. Math. 14 (2014) 461-472.

[25] R.S. Falk, Nonconforming finite element methods for the equations of linear elasticity. Math. Comput. 57 (1991) 529-550.

[26] M. Fortin, A three-dimensional quadratic nonconforming element. Numer. Math. 46 (1985) 269-279.

[27] M. Fortin and M. Soulie, A non-conforming piecewise quadratic finite element on triangles. Int. J. Numer. Methods Eng. 19 (1983) 505-520.

[28] T. Gudi, A new error analysis for discontinuous finite element methods for linear elliptic problems. Math. Comput. 79 (2010) 2169-2189.

[29] J.G. Heywood and R. Rannacher, Finite element approximation of the nonstationary Navier-Stokes problem. I. Regularity of solutions and second-order error estimates for spatial discretization. SIAM J. Numer. Anal. 19 (1982) $275-311$.

[30] K. Lipnikov and G. Manzini, A high-order mimetic method on unstructured polyhedral meshes for the diffusion equation. Accepted for publication in J. Comput. Phys.

[31] K. Lipnikov, G. Manzini, F. Brezzi and A. Buffa, The mimetic finite difference method for 3D magnetostatics fields problems. J. Comput. Phys. 230 (2011) 305-328.

[32] K. Lipnikov, G. Manzini and M. Shashkov, Mimetic finite difference method. J. Comput. Phys. 257 (2014) $1163-1227$.

[33] L.D. Marini, An inexpensive method for the evaluation of the solution of the lowest order Raviart-Thomas mixed method. SIAM J. Numer. Anal. 22 (1985) 493-496.

[34] G. Matthies and L. Tobiska, Inf-sup stable non-conforming finite elements of arbitrary order on triangles. Numer. Math. 102 (2005) 293-309.

[35] D. Mora, G. Rivera and R. Rodriguez, A virtual element method for the Steklov eigenvalue problem. Math. Models Methods Appl. Sci. 25 (2015) 1421-1445.

[36] C. Ortner, Nonconforming finite-element discretization of convex variational problems. IMA J. Numer. Anal. 31 (2011) 847864.

[37] A. Palha, P.P. Rebelo, R. Hiemstra, J. Kreeft and M. Gerritsma, Physics-compatible discretization techniques on single and dual grids, with application to the Poisson equation of volume forms. J. Comput. Phys. 257 (2014) 1394-1422.

[38] R. Rannacher and S. Turek, Simple nonconforming quadrilateral Stokes element. Numer. Meth. Partial Differ. Equ. 8 (1992) $97-111$. 
[39] G. Stoyan and A.E. Baran, Crouzeix-Velte decompositions for higher-order finite elements. Comput. Math. Appl. 51 (2006) 967-986.

[40] G. Strang, Variational Crimes in the Finite Element Method. In The Mathematical Foundations of the Finite Element Method with Applications to Partial Differential Equations. Proc. of Sympos., Univ. Maryland, Baltimore, Md. Academic Press, New York (1972) 689-710.

[41] G. Strang and G.J. Fix, An analysis of the finite element method. Prentice-Hall Series in Automatic Computation. PrenticeHall Inc., Englewood Cliffs, N. J. (1973).

[42] R. Verfürth. A note on polynomial approximation in Sobolev spaces. ESAIM: M2AN 33 (1999) 715-719. 